\title{
Spontaneous Lorentz and diffeomorphism violation, massive modes, and gravity
}

\author{
Robert Bluhm, ${ }^{1}$ Shu-Hong Fung, ${ }^{1,2}$ and V. Alan Kostelecký ${ }^{3}$ \\ ${ }^{1}$ Physics Department, Colby College, Waterville, Maine 04901, USA \\ ${ }^{2}$ Physics Department, Chinese University of Hong Kong, Shatin, N.T., Hong Kong \\ ${ }^{3}$ Physics Department, Indiana University, Bloomington, Indiana 47405, USA
}

(Received 30 December 2007; published 21 March 2008)

\begin{abstract}
Theories with spontaneous local Lorentz and diffeomorphism violation contain massless NambuGoldstone modes, which arise as field excitations in the minimum of the symmetry-breaking potential. If the shape of the potential also allows excitations above the minimum, then an alternative gravitational Higgs mechanism can occur in which massive modes involving the metric appear. The origin and basic properties of the massive modes are addressed in the general context involving an arbitrary tensor vacuum value. Special attention is given to the case of bumblebee models, which are gravitationally coupled vector theories with spontaneous local Lorentz and diffeomorphism violation. Mode expansions are presented in both local and spacetime frames, revealing the Nambu-Goldstone and massive modes via decomposition of the metric and bumblebee fields, and the associated symmetry properties and gauge fixing are discussed. The class of bumblebee models with kinetic terms of the Maxwell form is used as a focus for more detailed study. The nature of the associated conservation laws and the interpretation as a candidate alternative to Einstein-Maxwell theory are investigated. Explicit examples involving smooth and Lagrange-multiplier potentials are studied to illustrate features of the massive modes, including their origin, nature, dispersion laws, and effects on gravitational interactions. In the weak static limit, the massive mode and Lagrange-multiplier fields are found to modify the Newton and Coulomb potentials. The nature and implications of these modifications are examined.
\end{abstract}

PACS numbers: 11.30.Cp, 04.50.-h, 11.30.Er, 12.60.-i

\section{INTRODUCTION}

In relativistic quantum field theory, the nature of the field modes associated with the spontaneous breaking of an internal symmetry is now standard lore. When a global internal symmetry is spontaneously broken, one or more massless modes called Nambu-Goldstone (NG) modes appear [1]. If instead the symmetry is local, the Higgs mechanism can occur: the massless NG modes play the role of additional components of the gauge fields, which then propagate as massive modes [2]. In either case, the spontaneous symmetry breaking is typically driven by a potential term $V$ in the Lagrange density. The vacuum field configuration lies in a minimum $V_{0}$ of $V$. The massless NG modes can be understood as field excitations about the vacuum that preserve the value $V_{0}$, and they are associated with the broken generators of the symmetry. For many potentials, there are also additional excitations involving other values of $V$. These excitations, often called Higgs modes, correspond to additional massive modes that are distinct from any massive gauge fields.

This standard picture changes when the spontaneous breaking involves a spacetime symmetry rather than an internal one. In this work, we focus on spontaneous breaking of Lorentz and diffeomorphism symmetries, for which the corresponding Higgs mechanisms exhibit some unique features [3]. Spontaneous Lorentz violation occurs when one or more Lorentz-nonsinglet field configurations acquire nonzero vacuum expectation values. The field configurations of interest can include fundamental vectors or tensors, derivatives of scalars and other fields, and Lorentz- nonsinglet composites. The nonzero vacuum values are manifest both on the spacetime manifold and in local frames [4]. Their origin in spontaneous violation implies both local Lorentz violation and diffeomorphism violation, along with the existence of NG modes [5].

At the level of an underlying Planck-scale theory, numerous proposals exist that involve spontaneous Lorentz violation, including ones based on string theory [6], noncommutative field theories [7], spacetime-varying fields [8], quantum gravity [9], random-dynamics models [10], multiverses [11], braneworld scenarios [12], supersymmetry [13], and massive gravity [14-16]. At experimentally accessible scales, the observable signals resulting from Lorentz breaking can be described using effective field theory [17]. The general realistic effective field theory containing the Lagrange densities for both general relativity and the standard model along with all scalar terms involving operators for Lorentz violation is called the standard-model extension (SME) $[4,18]$. Searches for low-energy signals of Lorentz violation represent a promising avenue of investigation involving the phenomenology of quantum gravity [19]. Numerous experimental measurements of SME coefficients for Lorentz violation have already been performed [20], including ones with photons [21], electrons [22-24], protons and neutrons [25-27], mesons [28], muons [29], neutrinos [30], the Higgs [31], and gravity $[32,33]$.

For spontaneous Lorentz and diffeomorphism breaking, a general analysis of the nature of the NG modes and Higgs mechanisms is provided in Ref. [5]. One result is that the 
spontaneous breaking of local Lorentz symmetry implies spontaneous breaking of diffeomorphism symmetry and vice versa. Since six local Lorentz transformations and four diffeomorphisms can be broken, up to ten NG modes can appear. To characterize these, it is natural to adopt the vierbein formalism [34], in which the roles of local Lorentz transformations and diffeomorphisms are cleanly distinguished. It turns out that the vierbein itself naturally incorporates all ten modes. In an appropriate gauge, the six Lorentz NG modes appear in the antisymmetric components of the vierbein, while the four diffeomorphism NG modes appear along with the usual gravitational modes in the symmetric components.

The dynamical behavior of the various NG modes is determined by the structure of the action [5]. In a Lagrange density formed from tensor quantities and with diffeomorphism-covariant kinetic terms, the diffeomorphism NG modes are nonpropagating. This feature, unique to diffeomorphism symmetry, can be viewed as arising because the diffeomorphism NG field excitations that preserve $V_{0}$ include metric excitations, and the combined excitations cancel at propagation order in covariant derivatives and curvature. In contrast, the number of propagating Lorentz NG modes is strongly model dependent. For example, choosing the kinetic terms in the Lagrange density of the original field theory to eliminate possible ghost modes can also prevent the propagation of one or more Lorentz NG modes, leaving them instead as auxiliary fields.

Several types of possible Higgs mechanisms can be distinguished when spacetime symmetries are spontaneously broken. These have features distinct from the conventional Higgs mechanism of gauge field theories [2] and Higgs mechanisms involving gravity without Lorentz violation [35]. The analysis of Higgs mechanisms can be performed either using the vierbein formalism, which permits tracking of Lorentz and diffeomorphism properties, or by working directly with fields on the spacetime manifold. The results of both approaches are equivalent.

For local Lorentz symmetry, the role of the gauge fields in the vierbein formalism is played by the spin connection. The Lorentz Higgs mechanism occurs when the Lorentz NG modes play the role of extra components of the spin connection [5]. Some components of the spin connection then acquire mass via the covariant derivatives in the kinetic part of the Lagrange density. Explicit models displaying the Lorentz Higgs mechanism are known. For this mechanism to occur, the components of the spin connection must propagate as independent degrees of freedom, which requires a theory based on Riemann-Cartan geometry. If instead the theory is based on Riemann geometry, like general relativity, the spin connection is fixed nonlinearly in terms of the vierbein and its derivatives. The presence of these derivatives ensures that no mass terms emerge from the kinetic part of the Lagrange density, although the vierbein propagator is modified.
In the context of diffeomorphism symmetry, the role of the gauge fields is played by the metric. For spontaneous diffeomorphism breaking in Riemann spacetime, a conventional diffeomorphism Higgs mechanism cannot generate a mass for the graviton because the connection and hence the analogue of the usual mass term involve derivatives of the metric [3]. Also, since diffeomorphism NG modes are nonpropagating in a Lagrange density with covariant kinetic terms for reasons mentioned above, the propagating NG degrees of freedom required to generate massive fields via a conventional diffeomorphism Higgs mechanism are lacking.

In a conventional gauge theory with a nonderivative potential $V$, the gauge fields are absent from $V$ and so the potential cannot directly contribute to the gauge masses. However, in spontaneous Lorentz and diffeomorphism violation, massive Higgs-type modes involving the vierbein can arise via the alternative Higgs mechanism, which involves the potential $V$ [3]. The key point is that $V$ contains both tensor and metric fields, so field fluctuations about the vacuum value $V_{0}$ can contain quadratic mass terms involving the metric.

In this work, we study the nature and properties of the additional massive Higgs-type modes arising from this alternative Higgs mechanism. A general treatment is provided for a variety of types of potentials $V$ in gravitationally coupled theories with Riemann geometry. We investigate the effects of the massive modes on the physical properties of gravity. In certain theories with spontaneous Lorentz violation, the NG modes can play the role of photons [5], and we also examine the effects of the massive modes on electrodynamics in this context.

The next section of this work provides a general discussion of the origin and basic properties of the massive Higgs-type modes. Section III analyzes the role of these modes in vector theories with spontaneous Lorentz violation, known as bumblebee models. In Sec. IV, the massive modes in a special class of bumblebee models are studied in more detail for several choices of potential in the Lagrange density, and their effects on both the gravitational and electromagnetic interactions are explored. Section V summarizes our results. Some details about transformation laws are provided in the appendix. Throughout this work, the conventions and notations of Refs. [4,5] are used.

\section{MASSIVE MODES}

The characteristics of the massive Higgs-type modes that can arise from the alternative Higgs mechanism depend on several factors. Among them are the type of field configuration acquiring the vacuum expectation value and the form of the Lagrange density, including the choice of potential $V$ inducing spontaneous breaking of Lorentz and diffeomorphism symmetries. In this section, we outline some generic features associated with the alternative 
Higgs mechanism in a theory of a general tensor field $T_{\lambda \mu \nu} \ldots$ in a Riemann spacetime with metric $g_{\mu \nu}$. We consider first consequences of the choice of potential $V$, then discuss properties of vacuum excitations, and finally offer comments on the massive modes arising from the alternative Higgs mechanism.

\section{A. Potentials}

The potential $V$ in the Lagrange density is taken to trigger a nonzero vacuum expectation value

$$
\left\langle T_{\lambda \mu \nu \ldots}\right\rangle=t_{\lambda \mu \nu \cdots}
$$

for the tensor field, thereby spontaneously breaking local Lorentz and diffeomorphism symmetries. In general, $V$ varies with $T_{\lambda \mu \nu \ldots}$, its covariant derivatives, the metric $g_{\mu \nu}$, and possibly other fields. However, for simplicity we suppose here that $V$ has no derivative couplings and involves only $T_{\lambda \mu \nu} \ldots$ and $g_{\mu \nu}$. We also suppose that $V$ is everywhere positive except at its degenerate minima, which have $V=0$ and are continuously connected via the broken Lorentz and diffeomorphism generators. The vacuum is chosen to be the particular minimum in which $T_{\lambda \mu \nu} \ldots$ attains its nonzero value (1).

Since the Lagrange density is an observer scalar, $V$ must depend on fully contracted combinations of $T_{\lambda \mu \nu \cdots}$ and $g_{\mu \nu}$. Provided $T_{\lambda \mu \nu} \ldots$ has finite rank, the number of independent scalar combinations is limited. For example, for a symmetric two-tensor field $C_{\mu \nu}$ there are four independent possibilities, which can be given explicitly in terms of traces of powers of $C_{\mu \nu}$ and $g_{\mu \nu}$ [36]. It is convenient to denote generically these scalar combinations as $X_{m}$, where $m=1,2, \ldots, M$ ranges over the number $M$ of independent combinations. The functional form of the potential therefore takes the form

$$
V=V\left(X_{1}, X_{2}, \ldots, X_{M}\right)
$$

in terms of the scalars $X_{m}$.

The definition of the scalars $X_{m}$ can be chosen so that $X_{m}=0$ in the vacuum for all $m$. For example, a choice involving a quadratic combination of the tensor with zero vacuum value is

$$
X=T_{\lambda \mu \nu \cdots} g^{\lambda \alpha} g^{\mu \beta} g^{\nu \gamma} \cdots T_{\alpha \beta \gamma} \pm \pm t^{2},
$$

with $t^{2}$ the norm

$$
t^{2}=\mp t_{\lambda \mu \nu \cdots}\left\langle g^{\lambda \alpha}\right\rangle\left\langle g^{\mu \beta}\right\rangle\left\langle g^{\nu \gamma}\right\rangle \cdots t_{\alpha \beta \gamma \cdots}
$$

where $\left\langle g^{\lambda \alpha}\right\rangle$ is the vacuum value of the inverse metric. The $\mp$ sign is introduced for convenience so that $t^{2}$ can be chosen non-negative. In principle, $t^{2}$ could vary with spacetime position, which would introduce explicit spacetime-symmetry breaking, but it suffices for present purposes to take $t^{2}$ as a real non-negative constant.

The $M$ conditions $X_{m}=0$ fix the vacuum value $t_{\lambda \mu \nu} \ldots$ If only a subset of $N$ of these $M$ conditions is generated in a given theory, then the value of $t_{\lambda \mu \nu^{\cdots}}$ is specified up to $(M-N)$ coset transformations, and so the vacuum is degenerate under $(M-N)$ additional gauge symmetries. Note that these $(M-N)$ freedoms are distinct from Lorentz and diffeomorphism transformations.

It is useful to distinguish two classes of potentials $V$. The first consists of smooth functionals $V$ of $X_{m}$ that are minimized by the conditions $X_{m}=0$ for at least some $m$. These potentials therefore satisfy $V=V_{m}^{\prime}=0$ in the vacuum, where $V_{m}^{\prime}$ denotes the derivative with respect to $X_{m}$, and they fix the vacuum value of $T_{\lambda \mu \nu} \ldots$ to $t_{\lambda \mu \nu} \ldots$ modulo possible gauge freedoms. A simple example with the quantity $X$ in Eq. (3) is

$$
V_{S}(X)=\frac{1}{2} \kappa X^{2},
$$

where $\kappa$ is a constant. In this case, the vacuum value $t_{\lambda \mu \nu} \ldots$ is a solution of $V=V^{\prime}=0$, where $V^{\prime}$ denotes a derivative with respect to $X$. If the matrix $V_{m n}^{\prime \prime}$ of second derivatives has nonzero eigenvalues, the smooth functionals $V$ can give rise to quadratic mass terms in the Lagrange density involving the tensor and metric fields. Potentials $V$ of this type are therefore associated with the alternative Higgs mechanism, and they are the primary focus of our attention.

A second class of potentials introduces Lagrangemultiplier fields $\lambda_{m}$ for at least some $m$, to impose directly the conditions $X_{m}=0$ as constraint terms in the Lagrange density. We consider here both linear and quadratic functional forms for these constraints. An explicit linear example is

$$
V_{L}(\lambda, X)=\lambda X,
$$

while a quadratic one is

$$
V_{Q}(\lambda, X)=\frac{1}{2} \lambda X^{2}
$$

In each example, $\lambda$ is a Lagrange-multiplier field and has equation of motion with solution $X=0$, so the value $t_{\lambda \mu \nu}$. is a vacuum solution for the tensor. Potentials in the Lagrange-multiplier class are unlikely to be physical in detail because they enforce singular slicings in the phase space for the fields. However, when used with care they can be useful as limiting approximations to potentials in the smooth class, including those inducing the alternative Higgs mechanism [3]. Note that positivity of the potentials can constrain the range of the Lagrange multiplier field. For example, the off-shell value of $\lambda$ in $V_{L}$ must have the same sign as $X$, while that of $\lambda$ in $V_{Q}$ must be non-negative.

\section{B. Excitations}

Field excitations about the vacuum solution (1) can be classified in five types: gauge modes, NG modes, massive modes, Lagrange-multiplier modes, or spectator modes. Gauge modes arise if the potential $V$ fixes only $(M-N)$ of the $M$ conditions $X_{m}=0$, so that the vacuum is unspe- 
cified up to $N$ conditions. These modes can be disregarded for most purposes here because they can be eliminated via gauge fixing without affecting the physics. The NG modes are generated by the virtual action of the broken Lorentz and diffeomorphism generators on the symmetry-breaking vacuum. They preserve the vacuum condition $V=0$. Massive modes are excitations for which the symmetry breaking generates quadratic mass terms. Lagrangemultiplier modes are excitations of the Lagrange multiplier field. Finally, spectator modes are the remaining modes in the theory.

For smooth potentials, field excitations preserving the conditions $X_{m}=0$ for all $m$ have potential $V=0$. They also satisfy $V_{m}^{\prime}=0$. The NG modes are of this type. Excitations with $X_{m} \neq 0$ having nonzero potential $V \neq 0$ and $V_{m}^{\prime} \neq 0$ are massive modes, with mass matrix determined by the second derivatives $V_{m n}^{\prime \prime}$. Since the smooth potentials depend on the tensor $T_{\lambda \mu \nu} \ldots$ and the metric $g_{\mu \nu}$, the corresponding massive modes involve combinations of excitations of these fields.

In contrast, for Lagrange-multiplier potentials the conditions $X_{m}=0$ always hold on shell, which implies $V=0$ for all on-shell excitations. If also $V_{m}^{\prime}=0$, then the excitations remain in the potential minimum and include the NG modes. For linear functional forms of $V$, it follows that $V_{m}^{\prime}=\lambda_{m}$, so $V_{m}^{\prime}$ is nonzero only when the Lagrangemultiplier field $\lambda_{m}$ is excited. Any excitations of $T_{\lambda \mu \nu} \ldots$ and $g_{\mu \nu}$ must have $V=V_{m}^{\prime}=0$. For quadratic functional forms of $V$, one finds $V_{m}^{\prime}=0$ for all excitations, including the $\lambda$ field. We therefore can conclude that the combinations of $T_{\lambda \mu \nu \cdots}$ and $g_{\mu \nu}$ playing the role of massive modes for smooth potentials are constrained to zero for Lagrangemultiplier potentials. Evidently, studies of the alternative Higgs mechanism must be approached with care when the Lagrange-multiplier approximation to a smooth potential is adopted.

The tensor excitations about the vacuum can be expressed by expanding $T_{\lambda \mu \nu} \ldots$ as

$$
T_{\lambda \mu \nu \cdots}=t_{\lambda \mu \nu \cdots}+\tau_{\lambda \mu \nu \cdots}
$$

where the excitation $\tau_{\lambda \mu \nu} \ldots$ is defined as the difference $\tau_{\lambda \mu \nu \ldots} \equiv \delta T_{\lambda \mu \nu \ldots}=T_{\lambda \mu \nu \cdots}-t_{\lambda \mu \nu \ldots}$ between the tensor and its vacuum value. We also expand the metric

$$
g_{\mu \nu}=\left\langle g_{\mu \nu}\right\rangle+h_{\mu \nu}
$$

in terms of the metric excitations $h_{\mu \nu}$ about the metric background value $\left\langle g_{\mu \nu}\right\rangle$. For simplicity and definiteness, in much of what follows we take the background metric to be that of Minkowski spacetime, $\left\langle g_{\mu \nu}\right\rangle=\eta_{\mu \nu}$. We also suppose $t_{\lambda \mu \nu \ldots}$ is constant in this background, so that $\partial_{\alpha} t_{\lambda \mu \nu} \ldots=0$ in cartesian coordinates.

Other choices can be made for the expansion of the tensor about its vacuum value. One alternative is to expand the contravariant version of the tensor as

$$
T^{\lambda \mu \nu \cdots}=\bar{t}^{\lambda \mu \nu \cdots}+\tilde{T}^{\lambda \mu \nu \cdots} .
$$

In a Minkowski background, the vacuum values in the two expansions (8) and (10) are related simply by

$$
\bar{t}^{\lambda \mu \nu \cdots}=\eta^{\lambda \alpha} \eta^{\mu \beta} \eta^{\nu \gamma} \cdots t_{\alpha \beta \gamma} \cdots
$$

However, the relationship between the two tensor excitations at leading order involves also the metric excitation:

$$
\begin{aligned}
\tilde{T}^{\lambda \mu \nu \cdots}= & \tau^{\lambda \mu \nu \cdots}-h^{\lambda \alpha} t_{\alpha}{ }^{\mu \nu \cdots}-h^{\mu \alpha} t_{\alpha}^{\lambda}{ }^{{ }^{\cdots} \cdots} \\
& -h^{\nu \alpha} t^{\lambda \mu}{ }_{\alpha} \cdots-\ldots
\end{aligned}
$$

In this expression, indices have been raised using the Minkowski metric.

Any Lagrange multipliers $\lambda_{m}$ in the theory can also be expanded about their vacuum values $\bar{\lambda}_{m}$ as

$$
\lambda_{m}=\bar{\lambda}_{m}+\tilde{\lambda}_{m}
$$

For linear Lagrange-multiplier potentials, the equations of motion for $T_{\lambda \mu \nu} \ldots$ and $g_{\mu \nu}$ provide constraints on the vacuum values $\bar{\lambda}_{m}$. In a Minkowski background and a potential yielding $X_{m}=0$ for all $m$, the equations of motion for $T_{\lambda \mu \nu} \ldots$ can be solved to give

$$
\bar{\lambda}_{m}=0 .
$$

For quadratic Lagrange-multiplier potentials, the $\lambda_{m}$ are absent from the equations of motion. Their vacuum values are therefore physically irrelevant, and $\bar{\lambda}_{m}=0$ can also be adopted in this case. For the remainder of this work we take $\bar{\lambda}_{m}=0$, and for notational simplicity we write $\lambda_{m}$ for both the full field $\lambda_{m}$ and the excitation $\tilde{\lambda}_{m}$.

\section{Massive modes}

For a smooth potential $V$, the massive excitations typically involve a mixture of tensor and gravitational fields. As an example, consider the expression for $V_{S}$ in Eq. (5). This can be expanded in terms of the excitations $\tau_{\lambda \mu \nu} \ldots$ and $h_{\mu \nu}$. Retaining only terms up to quadratic order in a Minkowski background gives

$$
\begin{aligned}
V_{S} \approx & 2 \kappa\left[t ^ { \lambda \mu \nu \cdots } \left(\tau_{\lambda \mu \nu \cdots}-\frac{1}{2} h_{\lambda \alpha} t^{\alpha}{ }_{\mu \nu \cdots}-\frac{1}{2} h_{\mu \beta} t_{\lambda}{ }^{\beta}{ }_{\nu \cdots}\right.\right. \\
& \left.\left.-\frac{1}{2} h_{\nu \gamma} t_{\lambda \mu}{ }^{\gamma} \ldots-\ldots\right)\right]^{2},
\end{aligned}
$$

where index contractions are performed with the Minkowski metric $\eta_{\mu \nu}$. Evidently, the massive excitations in this example involve linear combinations of $\tau_{\lambda \mu \nu} \ldots$ with contractions of $h_{\mu \nu}$ and $t_{\lambda \mu \nu} \ldots$

The explicit expressions for the massive modes can be modified by local Lorentz and diffeomorphism gauge fixing. The action is symmetric under 10 local Lorentz and diffeomorphism symmetries, so there are 10 possible gauge conditions. For an unbroken symmetry generator, the effects of a gauge choice are conventional. For a broken symmetry generator, a gauge choice fixes the location in field space of the corresponding NG mode. For example, 
suitable gauge choices can place all the NG modes in the vierbein [5]. These gauge choices also affect the form of the massive modes. They can be used to isolate some or all of the massive modes as components of either the gravitational or tensor fields by gauging other components to zero. Alternatively, the gauge freedom can be used to simplify the equations of motion, while the massive modes remain a mixture of the excitations $\tau_{\lambda \mu \nu} \ldots$ and $h_{\mu \nu}$.

The behavior of the massive modes depends on the form of the kinetic terms in the Lagrange density as well as the form of the potential $V$. A gravitational theory with a dynamical tensor field $T_{\lambda \mu \nu}$. has kinetic terms for both $g_{\mu \nu}$ and $T_{\lambda \mu \nu} \ldots$ and hence for both $h_{\mu \nu}$ and $\tau_{\lambda \mu \nu} \ldots$. Since in the alternative Higgs mechanism the potential $V$ acts only as a source of mass, the issue of whether the massive modes propagate dynamically depends on the structure of these kinetic terms. In particular, propagating massive modes can be expected only if the theory without the potential $V$ contains the corresponding propagating massless modes.

It is desirable that any propagating modes be unitary and ghost-free. To avoid unitarity issues with higher-derivative propagation, the kinetic term for the metric excitation $h_{\mu \nu}$ can be taken to emerge as usual from the Einstein-Hilbert action. In the absence of the potential $V$, this is also a ghost-free choice. For the tensor field, higher-derivative propagation can be avoided by writing the general kinetic term $\mathcal{L}_{\mathrm{K}}$ as a weighted sum of all scalar densities formed from quadratic expressions in $T_{\lambda \mu \nu} \ldots$ that involve two covariant derivatives. For example, one such scalar density is

$$
\mathcal{L}_{\mathrm{K}} \sim e T_{\lambda \mu \nu} \ldots D_{\alpha} D^{\alpha} T^{\lambda \mu \nu \cdots},
$$

where $e=\sqrt{-g}$ is the vierbein determinant. In the absence of the potential $V$, the ghost-free requirement places strong constraints on allowed forms of $\mathcal{L}_{\mathrm{K}}$ and typically involves gauge symmetry for the tensor. For a vector field, for example, the Maxwell action is the unique ghost-free combination in the Minkowski spacetime limit. In any case, since the massive modes are combinations of $h_{\mu \nu}$ and $\tau_{\lambda \mu \nu} \ldots$, it follows that ghost-free propagation is possible only if the kinetic terms for these combinations are ghost-free. Note that the potential $V$ may explicitly break the tensor gauge symmetry, so requiring ghost-free kinetic terms is by itself insufficient to ensure ghost-free massive modes.

Under the assumption of Lorentz invariance, the FierzPauli action involving quadratic terms for $h_{\mu \nu}$ is the unique ghost-free choice for a free massive spin-2 field [37]. However, when coupled to a covariantly conserved energy-momentum tensor, the small-mass limit of a massive spin-2 field includes modes that modify the gravitational bending of light in disagreement with observation [38]. This presents an obstacle to constructing a viable theory of massive gravity. One avenue of investigation that might permit evading this obstacle is to relax the assumption of Lorentz invariance. Spontaneous Lorentz violation in closed string theory has been proposed [14] as a mechanism that might lead to graviton mass terms. Models involving infrared modifications of gravity have also been proposed that involve spontaneous Lorentz violation with ghosts [15] and that have explicit Lorentz violations [16]. Explicit Lorentz violation is incompatible with Riemann and Riemann-Cartan geometries but may be compatible with Finsler or other geometries $[4,39]$ or may be viewed as an approximation to spontaneous violation. In the present context, the possibility exists that the massive modes from the alternative Higgs mechanism could evade the Veltman-van Dam-Zakharov constraint via their origin in spontaneous Lorentz violation and their nature as mixtures of gravitational and tensor modes. For example, the expansion (15) includes quadratic terms involving contractions of $h_{\mu \nu}$ with the vacuum value $t_{\lambda \mu \nu}$, so a model with suitable vacuum values and incorporating also a ghost-free propagator for the corresponding modes would describe a type of propagating massive gravity without conventional Fierz-Pauli terms.

In the alternative Higgs mechanism, the existence of propagating massive modes involving the metric can be expected to affect gravitational physics. Effects can arise directly from the modified graviton propagator and also from the massive modes acting as sources for gravitational interactions. The latter can be understood by considering the energy-momentum tensor $T^{\mu \nu}$ of the full theory, which can be found by variation of the action with respect to the metric $g_{\mu \nu}$. The contribution $T_{V}^{\mu \nu}$ to $T^{\mu \nu}$ arising from the potential $V$ is

$$
T_{V}^{\mu \nu}=-g^{\mu \nu} V+2 V_{m}^{\prime} \frac{\delta X_{m}}{\delta g_{\mu \nu}}
$$

where a sum on $m$ is understood. For the vacuum solution $X_{m}=0$, which satisfies $V=V_{m}^{\prime}=0, T_{V}^{\mu \nu}$ remains zero and the gravitational sourcing is unaffected. The same is true for excitations for which $V$ and $V_{m}^{\prime}$ both vanish, such as the NG modes. However, the massive modes arising from a smooth potential have nonzero $V$ and $V_{m}^{\prime}$ and can therefore act as additional sources for gravity. These contributions can lead to a variety of gravitational effects including, for example, modifications of the Newton gravitational potential in the weak-field limit, which could have relevance for dark matter, or cosmological features such as dark energy [4].

Note that nonpropagating modes can also have similar significant physical effects on gravitational properties. This possibility holds for any excitations appearing in $V$, whether they are physical, ghost, or Lagrange-multiplier modes. For example, any Lagrange-multiplier fields are auxiliary by construction and so the $\lambda_{m}$ excitations are nonpropagating. Nonetheless, for linear Lagrangemultiplier potentials these excitations can contribute to 
$T_{V}^{\mu \nu}$ even though $V=0$ because $V_{m}^{\prime}$ is nonvanishing. However, a theory with a quadratic Lagrange-multiplier potential has $T_{V}^{\mu \nu}=0$ and therefore leaves the gravitational sourcing unaffected.

\section{BUMBLEBEE MODELS}

In this section, we focus attention on the special class of theories in which the role of the tensor $T_{\lambda \mu \nu} \ldots$ is played by a vector $B_{\mu}$ that acquires a nonzero vacuum expectation value $b_{\mu}$. These theories, called bumblebee models, are among the simplest examples of field theories with spontaneous Lorentz and diffeomorphism breaking. In what follows, bumblebee models are defined, their properties under local Lorentz and diffeomorphism transformations are presented, their mode content is analyzed, and issues involving gauge fixing and alternative mode expansions are considered.

\section{A. Basics}

The action $S_{B}$ for a single bumblebee field $B_{\mu}$ coupled to gravity and matter can be written as

$S_{B}=\int d^{4} x \mathcal{L}_{B}=\int d^{4} x\left(\mathcal{L}_{g}+\mathcal{L}_{g B}+\mathcal{L}_{\mathrm{K}}+\mathcal{L}_{V}+\mathcal{L}_{\mathrm{M}}\right)$.

In Riemann spacetime, the pure gravitational piece $\mathcal{L}_{g}$ of the Lagrange density is usually taken to be the EinsteinHilbert term supplemented by the cosmological constant $\Lambda$. The gravity-bumblebee couplings are described by $\mathcal{L}_{g B}$, while $\mathcal{L}_{\mathrm{K}}$ contains the bumblebee kinetic terms and any self-interaction terms. The component $\mathcal{L}_{V}$ consists of the potential $V\left(B_{\mu}\right)$, including terms triggering the spontaneous Lorentz violation. Finally, $\mathcal{L}_{\mathrm{M}}$ involves the bumblebee coupling to the matter or other sectors in the model.

The forms of $\mathcal{L}_{g B}$ and $\mathcal{L}_{K}$ are complicated in the general case. However, if attention is limited to terms quadratic in $B_{\mu}$ involving no more than two derivatives, then only five possibilities exist. The Lagrange density $\mathcal{L}_{B}$ can then be written as

$$
\begin{aligned}
\mathcal{L}_{B}= & \frac{1}{16 \pi G} e(R-2 \Lambda)+\sigma_{1} e B^{\mu} B^{\nu} R_{\mu \nu}+\sigma_{2} e B^{\mu} B_{\mu} R \\
& -\frac{1}{4} \tau_{1} e B^{\mu \nu} B_{\mu \nu}+\frac{1}{2} \tau_{2} e D_{\mu} B_{\nu} D^{\mu} B^{\nu} \\
& +\frac{1}{2} \tau_{3} e D_{\mu} B^{\mu} D_{\nu} B^{\nu}-e V+\mathcal{L}_{\mathrm{M}}
\end{aligned}
$$

where $G$ is the Newton gravitational constant and the fieldstrength tensor $B_{\mu \nu}$ is defined as

$$
B_{\mu \nu}=\partial_{\mu} B_{\nu}-\partial_{\nu} B_{\mu}
$$

The five real parameters $\sigma_{1}, \sigma_{2}, \tau_{1}, \tau_{2}, \tau_{3}$ in Eq. (19) are not all independent. Up to surface terms, which leave unaffected the equations of motion from the action, the condition

$$
\begin{aligned}
& \int d^{4} x\left(e B^{\mu} B^{\nu} R_{\mu \nu}-\frac{1}{2} e B^{\mu \nu} B_{\mu \nu}\right. \\
& \left.+e D_{\mu} B_{\nu} D^{\mu} B^{\nu}-e D_{\mu} B^{\mu} D_{\nu} B^{\nu}\right)=0
\end{aligned}
$$

is identically satisfied, so only four of the corresponding five terms in $\mathcal{L}_{B}$ are independent. No term of the form $e B^{\mu} B_{\mu} R$ appears in the condition (21), so the parameter $\sigma_{2}$ remains unaffected while the four parameters $\sigma_{1}, \tau_{1}, \tau_{2}, \tau_{3}$ become linked. For example, the identity (21) implies that the action for the Lagrange density (19) with five nonzero parameters $\sigma_{1}, \sigma_{2}, \tau_{1}, \tau_{2}, \tau_{3}$ is equivalent to an action of the same form but with only four nonzero parameters $\sigma_{1}^{\prime}=$ $\sigma_{1}+\tau_{3}, \sigma_{2}^{\prime}=\sigma_{2}, \tau_{1}^{\prime}=\tau_{1}-2 \tau_{3}, \tau_{2}^{\prime}=\tau_{2}-2 \tau_{3}$, while $\tau_{3}^{\prime}=0$. Moreover, other factors may also constrain some of the five parameters. For example, certain models of the form $\mathcal{L}_{B}$ yield equations of motion that imply additional relationships among the parameters. Also, certain physical limits such as the restriction to Minkowski spacetime can limit the applicability of the condition (21). Some cases with specific parameter values may be of particular interest for reasons of physics, geometry, or simplicity, such as the models with $\tau_{1}=1, \tau_{2}=\tau_{3}=0$ considered below, or the model with $\sigma_{2}=-\sigma_{1} / 2$ for which the bumblebeecurvature coupling involves the Einstein tensor, $\mathcal{L}_{B} \supset$ $\sigma_{1} e B^{\mu} B^{\nu} G_{\mu \nu}$. Since the most convenient choice of parameters depends on the specific model and on the physics being addressed, it is useful to maintain the five-parameter form (19) for generality.

Following the discussion in Sec. II A, we suppose the potential $V$ in Eq. (19) has no derivative couplings and is formed from scalar combinations of the bumblebee field $B_{\mu}$ and the metric $g_{\mu \nu}$. Only one independent scalar exists. It can be taken as the bumblebee version of $X$ in Eq. (3):

$$
X=B_{\mu} g^{\mu \nu} B_{\nu} \pm b^{2},
$$

where $b^{2}$ is a real non-negative constant. The potential $V(X)$ itself can be smooth in $X$, like the form (5), or it can involve Lagrange multipliers like the form (6) or (7). In any case, the vacuum is determined by the single condition

$$
X=B_{\mu} g^{\mu \nu} B_{\nu} \pm b^{2}=0 .
$$

In the vacuum, the potential vanishes, $V(X)=0$, and the fields $B_{\mu}, g_{\mu \nu}$ acquire vacuum values

$$
B_{\mu} \rightarrow\left\langle B_{\mu}\right\rangle=b_{\mu}, \quad g_{\mu \nu} \rightarrow\left\langle g_{\mu \nu}\right\rangle .
$$

The nonzero value $b_{\mu}$, which obeys $b_{\mu}\left\langle g^{\mu \nu}\right\rangle b_{\nu}=\mp b^{2}$, spontaneously breaks both Lorentz and diffeomorphism symmetry. Note that the choice of the potential $V$ can also have implications for the parameters in Eq. (19). For example, if the potential takes the quadratic Lagrangemultiplier form (7), then $\sigma_{2}$ can be taken as zero because a nonzero value merely acts to rescale $G$ and $\Lambda$. However, a nonzero value of $\sigma_{2}$ can have nontrivial consequences for 
models with other potentials, such as the smooth quadratic form (5).

For generic choices of parameters, the Lagrange density (19) is unitary because no more than two derivatives appear. However, as discussed in Sec. II C, the indefinite metric and generic absence of gauge invariance typically imply the presence of ghosts and corresponding negativeenergy problems, which can tightly constrain the viability of various models [40]. If the gravitational couplings and the potential $V$ are disregarded, there is a unique set of parameters ensuring the absence of ghosts: $\tau_{1}=1, \tau_{2}=$ $\tau_{3}=0$. With this choice, the kinetic term for the bumblebee becomes the Maxwell action, in which the usual U(1) gauge invariance excludes ghosts. When the gravitational terms and couplings are included, this gauge invariance is maintained for the parameter choice $\tau_{1}=1, \tau_{2}=\tau_{3}=$ $\sigma_{1}=\sigma_{2}=0$. The further inclusion of the potential $V$ breaks the U(1) gauge symmetry, but the form of the kinetic term ensures a remnant constraint on the equations of motion arising from the identity

$$
D_{\mu} D_{\nu} B^{\mu \nu} \equiv 0 .
$$

The action for these bumblebee models, introduced in Ref. [3], is therefore of particular interest. The corresponding Lagrange density

$$
\mathcal{L}_{\mathrm{KS}}=\frac{1}{16 \pi G} e(R-2 \Lambda)-\frac{1}{4} e B^{\mu \nu} B_{\mu \nu}-e V+\mathcal{L}_{\mathrm{M}},
$$

is investigated in more detail in Sec. IV. The reader is warned that some confusion about the relationship between these models and ones with nonzero $\tau_{2}$ and $\tau_{3}$ exists in the literature. In particular, results for the models (26) can differ from those obtained in models with nonzero $\tau_{2}, \tau_{3}$ via straightforward adoption of the limit $\tau_{2}, \tau_{3} \rightarrow 0$, due to the emergence of the remnant constraint (25).

The discussion above defines bumblebee models on the spacetime manifold, without introducing a local Lorentz basis. In this approach, the Lorentz NG modes are hidden within the bumblebee field $B_{\mu}$. Adopting instead a vierbein formulation reveals explicitly the local Lorentz properties of the models, and it also provides a natural way to incorporate spinor fields in the matter Lagrange density $\mathcal{L}_{\mathrm{M}}$. The vierbein $e_{\mu}{ }^{a}$ converts tensors expressed in a local basis to ones on the spacetime manifold. The spacetime metric $g_{\mu \nu}$ is related to the Minkowski metric $\eta_{a b}$ in the local frame as

$$
g_{\mu \nu}=e_{\mu}^{a} e_{\nu}^{b} \eta_{a b},
$$

while the bumblebee spacetime vector $B_{\mu}$ is related to the local bumblebee vector $B_{a}$ as

$$
B_{\mu}=e_{\mu}{ }^{a} B_{a} .
$$

A complete treatment in the vierbein formalism involves also introducing the spin connection $\omega_{\mu}^{a b}$, which appears in covariant derivatives acting on local quantities. In Riemann-Cartan geometry, where the spacetime has both curvature and torsion, the spin connection represents degrees of freedom independent of the vierbein. However, experimental constraints on torsion are tight [41]. In this work, we restrict attention to Riemann geometry, for which the torsion vanishes and the spin connection is fixed in terms of the vierbein. It therefore suffices for our purposes to consider the vierbein degrees of freedom and their role relative to the gravitational and NG modes. Bumblebee models in the more general context of Riemann-Cartan spacetime with nonzero torsion are investigated in Refs. [4,5].

There is a substantial literature concerning theories of vacuum-valued vectors coupled to gravity. The fiveparameter Lagrange density (19) excluding the potential $V$ and the cosmological constant was investigated by Will and Nordtvedt in the context of vector-tensor models of gravity [42]. Kostelecký and Samuel (KS) [3] introduced the potential $V$ triggering spontaneous Lorentz violation and studied both the smooth quadratic potential (5) and the linear Lagrange-multiplier case (6) for the class of models given by Eq. (26). The presence of the potential introduces a variety of qualitatively new effects, including the necessary breaking of U(1) gauge invariance [4], the existence of massless NG modes and massive modes [5], and potentially observable novel effects for post-newtonian physics [33] and for the matter sector [43]. The potential $V$ also leads to a candidate alternative description of the photon [5] and the graviton [4,36]. In Minkowski spacetime, more general potentials $V$ of hypergeometric form are known to satisfy the one-loop exact renormalization group [44]. Models of the form (19) with $\sigma_{1}=\sigma_{2}=0$, a unit timelike $b_{\mu}$, a linear Lagrange-multiplier potential $V_{L}$, and an additional fourth-order interaction for $B_{\mu}$ have been studied in some detail as possible unconventional theories of gravity $[45,46]$. Other works involving bumblebee models include Refs. [40,47-51].

An aspect of bumblebee models of particular interest is the appearance of massless propagating vector modes. This feature suggests the prospect of an alternative to the usual description of photons via U(1) gauge theory. The central idea is to identify the photon modes with the NG modes arising from spontaneous Lorentz violation. Early discussions along these lines centered on reinterpretating the photon or electron in the context of special relativity without physical Lorentz violation [52,53]. More recently, the Lorentz NG modes in certain bumblebee models with physical Lorentz violation have been shown to obey the Einstein-Maxwell equations in Riemann spacetime in axial gauge [5]. These models are further considered below, with the discussion initiated in Sec. IVA 3. One motivation of the present work is to investigate the role of massive modes and Lagrange-multiplier fields in this context. In particular, it is of interest to investigate possible modifications to 
electrodynamics and gravity, with an eye to novel phenomenological applications of spontaneous Lorentz and diffeomorphism breaking.

\section{B. Transformations}

In considering theories with violations of Lorentz and diffeomorphism symmetry, it is important to distinguish between observer and particle transformations [18]. Under either an observer general coordinate transformation or an observer local Lorentz transformation, geometric quantities such as scalars, vectors, tensors, and their derivatives remain unchanged, but the coordinate basis defining their components transforms. In contrast, particle transformations can change geometric quantities, independently of any coordinate system and basis.

In theories without spacetime-symmetry breaking, the component forms of the transformation laws for particle and observer transformations are inversely related. For example, under infinitesimal particle diffeomorphisms described by four infinitesimal displacements $\xi^{\mu}$, the components of the bumblebee field transform according to the Lie derivative as

$$
\begin{aligned}
& B_{\mu} \rightarrow B_{\mu}-\left(\partial_{\mu} \xi^{\nu}\right) B_{\nu}-\xi^{\nu} \partial_{\nu} B_{\mu}, \\
& B^{\mu} \rightarrow B^{\mu}+\left(\partial_{\nu} \xi^{\mu}\right) B^{\nu}-\xi^{\nu} \partial_{\nu} B^{\mu},
\end{aligned}
$$

while the metric transforms as

$$
\begin{aligned}
& g_{\mu \nu} \rightarrow g_{\mu \nu}-\left(\partial_{\mu} \xi^{\alpha}\right) g_{\alpha \nu}-\left(\partial_{\nu} \xi^{\alpha}\right) g_{\mu \alpha}-\xi^{\alpha} \partial_{\alpha} g_{\mu \nu}, \\
& g^{\mu \nu} \rightarrow g^{\mu \nu}+\left(\partial_{\alpha} \xi^{\mu}\right) g^{\alpha \nu}+\left(\partial_{\alpha} \xi^{\nu}\right) g^{\mu \alpha}-\xi^{\alpha} \partial_{\alpha} g^{\mu \nu} .
\end{aligned}
$$

Under infinitesimal observer general coordinate transformations, which are the observer equivalent of diffeomorphisms, the formulas for the transformations of the bumblebee and metric components take the same mathematical form up to a possible sign change in the arbitrary parameter $\xi^{\mu}$, even though these transformations are only the result of a change of coordinates. Similarly, under infinitesimal particle local Lorentz transformations with six parameters $\epsilon_{a b}=-\epsilon_{b a}$ related to the local Lorentz group element by $\Lambda_{a}{ }^{b} \approx \delta_{a}{ }^{b}+\epsilon_{a}{ }^{b}$, the local components of the bumblebee field transform as

$$
B_{a} \rightarrow B_{a}+\epsilon_{a}^{b} B_{b},
$$

with the formula for $B^{a}$ following from this by raising indices with $\eta^{a b}$. Under observer local Lorentz transformations, the corresponding transformation formulas again take the same mathematical form. However, any form of physical Lorentz and diffeomorphism breaking destroys the details of these equivalences.

A fundamental premise in classical physics is that the properties of a physical system are independent of the presence of a noninteracting observer. The observer is free to select a coordinate system to describe the physics of the system, but the physics cannot depend on this choice. In particular, this must remain true even when Lorentz and diffeomorphism symmetry are broken, whether explicitly or spontaneously. Viable candidate theories with spacetime-symmetry breaking must therefore be invariant under observer transformations, which are merely changes of coordinate system. For example, the SME is formulated as a general effective field theory that is invariant under observer general coordinate transformations and under observer local Lorentz transformations $[4,17,18]$.

In contrast, a theory with physical Lorentz and diffeomorphism breaking cannot by definition remain fully invariant under the corresponding particle transformations. For example, if the breaking is explicit, the action of the theory changes under particle transformations. If instead the breaking is spontaneous, the action remains invariant and the equations of motion transform covariantly, as usual. However, the vacuum solution to the equations of motion necessarily contains quantities with spacetime indices that are unchanged by particle transformations. These vacuum values and the excitations around them can lead to physical effects revealing the symmetry breaking.

In bumblebee models, the relevant spacetime vacuum values are those of the bumblebee and metric fields, denoted $\left\langle B_{\mu}\right\rangle$ and $\left\langle g_{\mu \nu}\right\rangle$. These are unaffected by particle diffeomorphisms, and their nonzero components thereby reveal the broken particle diffeomorphisms. In contrast, $\left\langle B_{\mu}\right\rangle$ and $\left\langle g_{\mu \nu}\right\rangle$ both transform as usual under observer general coordinate transformations, which therefore are unbroken. Analogous results hold for the local-frame vacuum value $\left\langle B_{a}\right\rangle$ of the local bumblebee field. The components $\left\langle B_{a}\right\rangle$ remain unaffected by particle local Lorentz transformations, with the invariance of the nonzero components resulting from the breaking of local Lorentz generators, while the components $\left\langle B_{a}\right\rangle$ transform under observer local Lorentz transformations in the usual way. Similarly, the vacuum value $\left\langle e_{\mu}{ }^{a}\right\rangle$ of the vierbein is unchanged by both particle diffeomorphisms and particle Lorentz transformations, but it transforms as a vector under the corresponding observer transformations.

By virtue of the relation (28) between the spacetime and local bumblebee fields, it follows that spontaneous local Lorentz violation is necessarily accompanied by spontaneous diffeomorphism violation and vice versa [5]. The point is that the vacuum value of the vierbein is nonzero, so the existence of a nonzero $\left\langle B_{a}\right\rangle$ spontaneously breaking local Lorentz symmetry also implies the existence of a nonzero $\left\langle B_{\mu}\right\rangle$ spontaneously breaking diffeomorphism symmetry, and vice versa. Note, however, that this result fails for explicit violation, where the analogue of the relation (28) is absent. In general, explicit local Lorentz violation occurs when a nonzero quantity $t_{a b c \ldots}$ is externally prescribed in the local frame, but the corresponding spacetime quantity $t_{\lambda \mu \nu \ldots} \equiv e_{\lambda}{ }^{a} e_{\mu}{ }^{b} e_{\nu}{ }^{c} \cdots t_{a b c \ldots .}$ is defined 
using the full vierbein and hence remains invariant under diffeomorphisms. Similarly, explicit diffeomorphism violation occurs when a nonzero quantity $t_{\lambda \mu \nu \ldots}$ is externally prescribed on the spacetime manifold, but the corresponding local quantity defined via the inverse vierbein remains invariant under local Lorentz transformations.

\section{Mode expansions}

To study the content and behavior of the modes, the fields can be expanded as infinitesimal excitations about their vacuum values. At the level of the Lagrange density, it suffices for most purposes to keep only terms to second order in the field excitations, which linearizes the equations of motion.

Assuming for simplicity a Minkowski background $\left\langle g_{\mu \nu}\right\rangle=\eta_{\mu \nu}$, we expand the metric and its inverse as

$$
g_{\mu \nu}=\eta_{\mu \nu}+h_{\mu \nu}, \quad g^{\mu \nu} \approx \eta^{\mu \nu}-h^{\mu \nu} .
$$

For the local bumblebee vector, we write

$$
B_{a}=b_{a}+\beta_{a},
$$

where the vacuum value $\left\langle B_{a}\right\rangle$ is denoted $b_{a}$ and assumed constant, and the infinitesimal excitations are denoted $\beta_{a}$. The vacuum condition (23) implies

$$
b_{a} \eta^{a b} b_{b}=\mp b^{2} .
$$

In a Minkowski background, the vierbein vacuum value can be chosen to be $\left\langle e_{\mu}{ }^{a}\right\rangle=\delta_{\mu}{ }^{a}$ in cartesian coordinates. The expansions of the vierbein and its inverse are

$$
\begin{aligned}
& e_{\mu}{ }^{a} \approx \delta_{\mu}{ }^{a}+\frac{1}{2} h_{\mu}{ }^{a}+\chi_{\mu}{ }^{a}, \\
& e^{\mu}{ }_{a} \approx \delta^{\mu}{ }_{a}-\frac{1}{2} h^{\mu}{ }_{a}+\chi^{\mu}{ }_{a},
\end{aligned}
$$

where $h_{\mu a}$ and $\chi_{\mu a}$ are, respectively, the symmetric and antisymmetric components of the vierbein. The covariant and contravariant components of the bumblebee field follow from Eq. (28) as

$$
\begin{aligned}
& B_{\mu} \approx \delta_{\mu}{ }^{a}\left(b_{a}+\beta_{a}\right)+\left(\frac{1}{2} h_{\mu}{ }^{a}+\chi_{\mu}{ }^{a}\right) b_{a}, \\
& B^{\mu} \approx \delta^{\mu}{ }_{a}\left(b^{a}+\beta^{a}\right)+\left(-\frac{1}{2} h^{\mu}{ }_{a}+\chi^{\mu}{ }_{a}\right) b^{a} .
\end{aligned}
$$

Since the local and spacetime background metrics are both Minkowski and since the excitations are infinitesimal, the distinction between the Latin local indices and the Greek spacetime indices can be dropped. We adopt Greek indices for most purposes that follow, raising and lowering indices on purely first-order quantities with $\eta^{\mu \nu}$ and $\eta_{\mu \nu}$. The vierbein expansions (35) can then be rewritten as

$$
\begin{aligned}
& e_{\mu \nu} \approx \eta_{\nu \sigma} e_{\mu}^{\sigma} \approx \eta_{\mu \nu}+\frac{1}{2} h_{\mu \nu}+\chi_{\mu \nu}, \\
& e^{\mu \nu} \approx \eta^{\nu \sigma} e^{\mu}{ }_{\sigma} \approx \eta^{\mu \nu}-\frac{1}{2} h^{\mu \nu}+\chi^{\mu \nu} .
\end{aligned}
$$

The vacuum value for the bumblebee vector becomes

$$
\left\langle B_{\mu}\right\rangle=\left\langle e_{\mu}{ }^{a}\right\rangle b_{a}=\delta_{\mu}{ }^{a} b_{a} \equiv b_{\mu} .
$$

It is convenient to decompose the local vector excitations $\beta_{\mu} \equiv \delta_{\mu}{ }^{a} \beta_{a}$ into transverse and longitudinal pieces with respect to $b_{\mu}$. Excluding for simplicity the case of lightlike $b_{\mu}$, we write

$$
\beta_{\mu}=\beta_{\mu}^{\mathrm{t}}+\beta \hat{b}_{\mu}, \quad \beta_{\mu}^{\mathrm{t}} b^{\mu}=0,
$$

where $\hat{b}_{\mu}=b_{\mu} / \sqrt{b^{2}}$ is a vector along the direction of $b_{\mu}$ obeying $\hat{b}^{\mu} \hat{b}_{\mu}=\mp 1$. Using this decomposition, the bumblebee mode expansions (36) become

$$
\begin{aligned}
& B_{\mu} \approx b_{\mu}+\left(\frac{1}{2} h_{\mu \nu}+\chi_{\mu \nu}\right) b^{\nu}+\beta_{\mu}^{\mathrm{t}}+\beta \hat{b}_{\mu}, \\
& B^{\mu} \approx b^{\mu}+\left(-\frac{1}{2} h^{\mu \nu}+\chi^{\mu \nu}\right) b_{\nu}+\beta^{\mathrm{t} \mu}+\beta \hat{b}^{\mu} .
\end{aligned}
$$

It is instructive to count degrees of freedom in the expressions (37) and (40). On the left-hand side, the vierbein has 16 components and the bumblebee field 4 , for a total of 20. On the right-hand side, the symmetric metric component $h_{\mu \nu}$ has 10, the antisymmetric component $\chi_{\mu \nu}$ has 6 , the transverse bumblebee excitation $\beta_{\mu}^{\mathrm{t}}$ has 3 , and the longitudinal excitation $\beta$ has 1 , producing the required matching total of 20 . Of these 20 degrees of freedom, 6 are metric modes, 4 are bumblebee modes, while 6 are associated with local Lorentz transformations and 4 with diffeomorphisms. The explicit transformations of all the field components under particle diffeomorphisms and local Lorentz transformations can be deduced from the full-field expressions and from the invariance of the vacuum values. A list of formulas for both particle and observer transformations is provided in the appendix.

Among all the excitations, only the longitudinal component $\beta$ of the bumblebee field is invariant under both particle diffeomorphisms and local Lorentz transformations. It is therefore a physical degree of freedom in any gauge. Moreover, using Eqs. (23) and (40) reveals that exciting the $\beta$ mode alone produces a nonzero value of $X$, given at first order by

$$
X=B_{\mu} g^{\mu \nu} B_{\nu} \pm b^{2} \approx 2\left(b^{\mu} \hat{b}_{\mu}\right) \beta .
$$

As a result, the excitation $\beta$ is associated with a nonminimal value of the potential, and it therefore cannot be an NG mode. In fact, for the case of a smooth quadratic potential, $\beta$ is a massive mode. In contrast, in a theory with a Lagrange-multiplier potential where the constraint $X=0$ is enforced as an equation of motion, the massive mode $\beta$ identically vanishes.

\section{Gauge fixing and NG modes}

Since the spacetime-symmetry breaking in bumblebee models is spontaneous, the four diffeomorphisms parametrized by $\xi_{\mu}$ and the six local Lorentz transformations parametrized by $\epsilon_{\mu \nu}$ leave invariant the bumblebee action (19) and transform covariantly the equations of motion. 
Fixing the gauge freedom therefore requires 10 gauge conditions.

For the diffeomorphism freedom, a choice common in the literature is the harmonic gauge

$$
\partial_{\mu} \bar{h}^{\mu \nu}=0,
$$

where $\bar{h}_{\mu \nu}=h_{\mu \nu}-\frac{1}{2} \eta_{\mu \nu} h$ and $\bar{h}=-h \equiv-h^{\mu}{ }_{\mu}$. In the harmonic gauge, the Einstein tensor becomes $G_{\mu \nu} \approx$ $-\frac{1}{2} \square \bar{h}_{\mu \nu}$ at linear order. An alternative choice for the diffeomorphism degrees of freedom is the axial gravitational gauge

$$
h_{\mu \nu} b^{\nu}=0 \text {. }
$$

Both these gauge choices represent four conditions. To fix the local Lorentz freedom, it is common to eliminate all six antisymmetric vierbein components by imposing the six conditions

$$
\chi_{\mu \nu}=0 .
$$

Other choices are possible here too. Consider, for example, the decomposition of $\chi_{\mu \nu}$ in terms of projections along $b_{\mu}$,

$$
\begin{aligned}
\chi_{\mu \nu} & =\chi_{\mu \nu}^{t t} \mp \chi_{\mu}^{t} \hat{b}_{\nu} \pm \chi_{\nu}^{t} \hat{b}_{\mu}, \\
\chi_{\mu \nu}^{t t} b^{\nu} & =\chi_{\nu}^{t} b^{\nu}=0,
\end{aligned}
$$

which is the analogue of Eq. (39) for $\beta_{\mu}$. The components $\chi_{\mu \nu}^{t t}$ and $\chi_{\mu}^{t} \equiv \chi_{\mu \nu} \hat{b}^{\nu}$ each contain 3 degrees of freedom. Inspection of the transformation laws shows that an alternative to fixing the local Lorentz gauge via Eq. (44) is the set of six conditions

$$
\chi_{\mu \nu}^{t t}=\beta_{\mu}^{t}=0 .
$$

Note, however, that the combination $\left(\chi_{\mu \nu} b^{\nu}+\beta_{\mu}^{\mathrm{t}}\right)$ appearing in $B_{\mu}$ in Eq. (40) is invariant and therefore cannot be gauged to zero. Evidently, the associated local Lorentz degrees of freedom must remain somewhere in the theory.

The bumblebee vacuum value $\left\langle B_{\mu}\right\rangle=b_{\mu}$ breaks one of the four diffeomorphism symmetries. The broken generator is associated with the projected component $\xi_{\nu} b^{\nu}$ of the parameter $\xi_{\mu}$. Analogously, the vacuum value $\left\langle B_{a}\right\rangle=b_{a}$ breaks three of the six local Lorentz symmetries. The broken generators are associated with the components $\epsilon_{a b} b^{b} \approx \epsilon_{\mu \nu} b^{\nu}$ of $\epsilon_{\mu \nu}$ projected along $b^{\nu}$. In each case, the unbroken generators are associated with the orthogonal complements to the projections.

Since the vacuum breaks one particle diffeomorphism and three local Lorentz transformations, four NG modes appear. A useful general procedure to identify these modes is first to make virtual particle transformations using the broken generators acting on the appropriate vacuum values for the fields in $V$, and then to promote the corresponding parameters $\epsilon_{\mu \nu}$ and $\xi_{\mu}$ to field excitations [5]:

$$
\xi_{\mu} \rightarrow \Xi_{\mu}, \quad \epsilon_{\mu \nu} \rightarrow \mathcal{E}_{\mu \nu}=-\mathcal{E}_{\nu \mu} .
$$

The properties of $\Xi_{\mu}$ and $\mathcal{E}_{\mu \nu}$ under various particle and observer transformations are given in the appendix. The projections $\xi_{\nu} b^{\nu}$ and $\epsilon_{\mu \nu} b^{\nu}$ associated with the broken generators determine the NG modes, which are therefore $\Xi_{\nu} b^{\nu}$ and $\mathcal{E}_{\mu \nu} b^{\nu}$.

We can follow this procedure to elucidate the relationship between the NG modes and the component fields in the decomposition (40) of $B_{\mu}$. Consider first the diffeomorphism NG mode, which is generated by a virtual transformation acting on the spacetime vacuum value $\left\langle B_{\mu}\right\rangle$ and involving the broken diffeomorphism generator. The relevant transformation is given in Eq. (A1), and it yields

$$
\left\langle B_{\mu}\right\rangle \rightarrow b_{\mu}-\left(\partial_{\mu} \Xi_{\nu}\right) b^{\nu} .
$$

Comparison of this result to the form of Eq. (40) reveals that the four vierbein combinations $\left(\frac{1}{2} h_{\mu \nu}+\chi_{\mu \nu}\right) b^{\nu}$ contain the diffeomorphism NG mode. This agrees with the result obtained by combining virtual diffeomorphisms on the vacuum values of the component fields in Eq. (40):

$$
\begin{aligned}
\left\langle h_{\mu \nu} b^{\nu}\right\rangle & \rightarrow-\left(\partial_{\mu} \Xi_{\nu}+\partial_{\nu} \Xi_{\mu}\right) b^{\nu}, \\
\left\langle\chi_{\mu \nu} b^{\nu}\right\rangle & \rightarrow-\frac{1}{2}\left(\partial_{\mu} \Xi_{\nu}-\partial_{\nu} \Xi_{\mu}\right) b^{\nu}, \\
\left\langle\beta_{\mu}^{t}\right\rangle & \rightarrow 0, \quad\langle\beta\rangle \rightarrow 0 .
\end{aligned}
$$

Note also that the combinations $\left(\frac{1}{2} h_{\mu \nu}+\chi_{\mu \nu}\right) b^{\nu}$ maintain the potential minimum $V=0$, as is expected for an NG mode.

In contrast, the three Lorentz NG modes $\mathcal{E}_{\mu \nu} b^{\nu}$ are generated by virtual transformations acting on the localframe vacuum value $\left\langle B_{a}\right\rangle$ and involving the broken local Lorentz generators:

$$
\left\langle B_{a}\right\rangle \rightarrow b_{a}+\mathcal{E}_{a b} b^{b},
$$

Comparison with Eqs. (33) and (39) shows that the transverse field $\beta_{\mu}^{t}$ contains the three Lorentz NG modes $\mathcal{E}_{\mu \nu} b^{\nu}$. Note that there are exactly 3 degrees of freedom in $\beta_{\mu}^{t}$, all of which maintain the potential minimum $V=0$. This result can be used to connect the component fields in the decomposition of $B_{\mu}$ with the three Lorentz NG modes $\mathcal{E}_{\mu \nu} b^{\nu}$. The vacuum value $\left\langle B_{\mu}\right\rangle$ itself is invariant under local Lorentz transformations, so another component field must also contain the Lorentz NG modes. Performing virtual local Lorentz transformations on the vacuum values of the component fields gives

$$
\begin{aligned}
\left\langle h_{\mu \nu} b^{\nu}\right\rangle & \rightarrow 0, \quad\left\langle\chi_{\mu \nu} b^{\nu}\right\rangle \\
\left\langle\beta_{\mu}^{t}\right\rangle & \rightarrow-\mathcal{E}_{\mu \nu} b_{\mu \nu} b^{\nu}, \quad\langle\beta\rangle
\end{aligned}
$$

which shows that the three combinations $\left\langle\chi_{\mu \nu} b^{\nu}\right\rangle$ also contain the three Lorentz NG modes.

Combining the results (49) and (51) reveals the following NG mode content for the component field combinations in the decomposition (40) of $B_{\mu}$ : 


$$
\begin{aligned}
\left(-\frac{1}{2} h_{\mu \nu}+\chi_{\mu \nu}\right) b^{\nu} & =-\left(\partial_{\mu} \Xi_{\nu}\right) b^{\nu}-\mathcal{E}_{\mu \nu} b^{\nu}, \\
\beta_{\mu}^{t} & =\mathcal{E}_{\mu \nu} b^{\nu}, \\
\beta & \supset 0 .
\end{aligned}
$$

We see that the four combinations $\left(\frac{1}{2} h_{\mu \nu}+\chi_{\mu \nu}\right) b^{\nu}$ contain a mixture of the diffeomorphism NG mode and the three Lorentz NG modes, while the three combinations $\beta_{\mu}^{t}$ contain only the three Lorentz NG modes.

By fixing the 10 gauge freedoms in the theory, the above results can be used to determine the physical content of the field $B_{\mu}$. Suppose first we adopt the 10 conditions (43) and (44). Then, the bumblebee field becomes

$$
\begin{aligned}
B_{\mu} & \approx b_{\mu}+\beta_{\mu}^{t}+\beta \hat{b}_{\mu} \\
& =b_{\mu}+\mathcal{E}_{\mu \nu} b^{\nu}+\beta \hat{b}_{\mu},
\end{aligned}
$$

where the result (52) has been used. Note that the fixed gauge means that fields with different transformation properties can appear on the left- and right-hand sides. In this gauge, the four components of $B_{\mu}$ are decomposed into three Lorentz NG modes associated with $\beta_{\mu}^{t}$ and the one massive mode $\beta$. The diffeomorphism NG mode is absent. It obeys

$$
\left(\partial_{\mu} \Xi_{\nu}\right) b^{\nu}=-\left(\partial_{\nu} \Xi_{\mu}\right) b^{\nu}
$$

and hence $b^{\mu}\left(\partial_{\mu} \Xi_{\nu}\right) b^{\nu}=0$, and it is locked to the Lorentz NG modes via the equation

$$
\left(\partial_{\mu} \Xi_{\nu}\right) b^{\nu}=-\mathcal{E}_{\mu \nu} b^{\nu} .
$$

For the diffeomorphism NG mode, this gauge is evidently analogous to the unitary gauge in a non-Abelian gauge theory.

An alternative gauge choice could be to impose the 10 conditions (43) and (46) instead. This gives

$$
\begin{aligned}
B_{\mu} & \approx b_{\mu}+\chi_{\mu}^{t}+\beta \hat{b}_{\mu} \\
& =b_{\mu}+\mathcal{E}_{\mu \nu} b^{\nu}+\beta \hat{b}_{\mu},
\end{aligned}
$$

where in this gauge the Lorentz NG modes $\mathcal{E}_{\mu \nu} b^{\nu}$ are identified with $\chi_{\mu}^{t}$ instead of $\beta_{\mu}^{t}$. One way to understand this identification is to perform a local Lorentz transformation with parameter $\epsilon_{\mu \nu}=-\mathcal{E}_{\mu \nu}$ on the first result in Eq. (53). This changes the value of $\beta_{\mu}^{t}$ from $\mathcal{E}_{\mu \nu} b^{\nu}$ to zero, while simultaneously converting $\chi_{\mu}^{t}$ from zero to $\chi_{\mu}^{t}=\mathcal{E}_{\mu \nu} b^{\nu}$. In this gauge, the explicit decomposition (56) of $B_{\mu}$ in terms of NG modes is the same as that in Eq. (53), but the three Lorentz NG modes are now associated with the components $\chi_{\mu}^{t}$ of the vierbein. The diffeomorphism mode remains absent. It still obeys the condition (54) and is locked to the Lorentz NG modes by Eq. (55).

Partial gauge conditions can also be imposed. For example, suppose the choice (46) is made for the local Lorentz gauge, while the diffeomorphism gauge remains unfixed. Then, the 4 degrees of freedom in the combination $\left(\frac{1}{2} h_{\mu \nu}+\chi_{\mu \nu}\right) b^{\nu}$ consist of the three Lorentz NG modes and the diffeomorphism NG mode, and all the NG modes are contained in the vierbein [5]. The bumblebee field can be written as

$$
B_{\mu} \approx b_{\mu}-\left(\partial_{\mu} \Xi_{\nu}\right) b^{\nu}+\mathcal{E}_{\mu \nu} b^{\nu}+\beta \hat{b}_{\mu} .
$$

Note that only one projection of $\Xi_{\nu}$ appears, even though four diffeomorphism choices remain. The corresponding expression for $B^{\mu}$ includes additional metric contributions and is given by

$$
B^{\mu} \approx b^{\mu}+\left(\partial_{\nu} \Xi^{\mu}\right) b^{\nu}+\mathcal{E}^{\mu \nu} b_{\nu}+\beta \hat{b}^{\mu} .
$$

This equation contains contributions from all four fields $\Xi_{\mu}$. However, if the diffeomorphism excitations are restricted only to the one for the broken generator, for which $\Xi_{\mu}$ obeys $b_{\mu} \Xi_{\nu}=b_{\nu} \Xi_{\mu}$, then $B^{\mu}$ also reduces to an expression depending only on the diffeomorphism NG mode $\Xi_{\nu} b^{\nu}$. Related results are obtained in Ref. [5], which investigates the fate of the NG modes using a decomposition of the vierbein into transverse and longitudinal components along $b_{\mu}$. This decomposition leads to the same relations as Eqs. (57) and (58) when the condition $b_{\mu} \Xi_{\nu}=$ $b_{\nu} \Xi_{\mu}$ is applied.

Even without gauge fixing, the fields $\Xi_{\mu}$ cancel at linear order in both $G_{\mu \nu}$ and $B_{\mu \nu}$. As a result, propagating diffeomorphism NG modes cannot appear. This is a special case of a more general result. By virtue of their origin as virtual particle transformations, the diffeomorphism NG modes appear as certain components of representation-irreducible fields with nonzero vacuum values. However, diffeomorphism invariance ensures these modes enter in the metric and bumblebee fields in combinations that cancel in a diffeomorphism-invariant action, including the general bumblebee action (19). In contrast, the Lorentz NG modes do play a role in the bumblebee action. They can be identified with a massless vector field $A_{\mu} \equiv \mathcal{E}_{\mu \nu} b^{\nu}$ in the fixed axial gauge $A_{\mu} b^{\mu} \approx 0$. The fully gauge-fixed expression (53) for $B_{\mu}$ then becomes

$$
B_{\mu} \approx b_{\mu}+A_{\mu}+\beta \hat{b}_{\mu},
$$

where the transverse components have the form of photon fields in the axial gauge, and the longitudinal mode is the massive mode $\beta$.

\section{E. Alternative expansions}

The analysis in the previous subsections is based on the local-frame expansion (33) of the bumblebee field $B_{a}$. However, other mode expansions are possible, including ones in which the vierbein makes no explicit appearance and the local Lorentz transformations are no longer manifest. This subsection offers a few comments on two alternative expansions used in some of the literature.

The first alternative mode expansion is specified using the covariant spacetime components of the bumblebee field 
$[5,17]$,

$$
B_{\mu}=b_{\mu}+\mathcal{E}_{\mu},
$$

where $\mathcal{E}_{\mu}$ represents the excitations of the spacetime bumblebee field around the vacuum value $b_{\mu}$. It follows that the contravariant components are

$$
B^{\mu} \approx b^{\mu}+\mathcal{E}^{\mu}-h^{\mu \nu} b_{\nu}
$$

These fields are linked to the vierbein and the local-frame fluctuations $\beta_{\mu}$ via

$$
\mathcal{E}_{\mu}=\left(\frac{1}{2} h_{\mu \nu}+\chi_{\mu \nu}\right) b^{\nu}+\beta_{\mu}^{\mathrm{t}}+\beta \hat{b}_{\mu} .
$$

The fields $\mathcal{E}_{\mu}$ are scalars under particle local Lorentz transformations, but transform under particle diffeomorphisms as

$$
\begin{aligned}
\mathcal{E}_{\mu} & \rightarrow \mathcal{E}_{\mu}-\left(\partial_{\mu} \xi_{\alpha}\right) b^{\alpha}, \\
\mathcal{E}^{\mu} \equiv \eta^{\mu \nu} \mathcal{E}_{\nu} & \rightarrow \mathcal{E}^{\mu}-\eta^{\mu \nu}\left(\partial_{\nu} \xi_{\alpha}\right) b^{\alpha} .
\end{aligned}
$$

Note that this usage of $\mathcal{E}^{\mu}$ differs from that in Ref. [5].

The second alternative expansion [33,45,47-49] starts instead with the contravariant bumblebee components

$$
B^{\mu}=b^{\mu}+\mathcal{E}^{\prime \mu},
$$

where a prime is used to distinguish the excitations $\mathcal{E}^{\prime \mu}$ from the previous case. The corresponding covariant components are then

$$
B_{\mu} \approx b_{\mu}+\mathcal{E}_{\mu}^{\prime}+h_{\mu \nu} b^{\nu} .
$$

The field redefinitions connecting these to the vierbein and previous case are

$$
\begin{aligned}
\mathcal{E}^{\prime \mu} & =\left(-\frac{1}{2} h^{\mu \nu}+\chi^{\mu \nu}\right) b_{\nu}+\beta_{\mathrm{t}}^{\mu}+\beta \hat{b}^{\mu} \\
& =\eta^{\mu \nu} \mathcal{E}_{\mu}-h^{\mu \nu} b_{\nu} .
\end{aligned}
$$

The fields $\mathcal{E}^{\prime \mu}$ are scalars under particle local Lorentz transformations but transform under particle diffeomorphisms as

$$
\begin{aligned}
\mathcal{E}^{\prime \mu} & \rightarrow \mathcal{E}^{\prime \mu}+\left(\partial_{\alpha} \xi^{\mu}\right) b^{\alpha}, \\
\mathcal{E}_{\mu}^{\prime} \equiv \eta_{\mu \nu} \mathcal{E}^{\prime \mu} & \rightarrow \mathcal{E}_{\mu}^{\prime}+\left(\partial_{\alpha} \xi_{\mu}\right) b^{\alpha} .
\end{aligned}
$$

Note that the fields $\mathcal{E}_{\mu}$ and $\mathcal{E}_{\mu}^{\prime}$ have different transformation properties and contain different mixes of the bumblebee and metric excitations. However, $\mathcal{E}_{\mu}$ and $\mathcal{E}_{\mu}^{\prime}$ take the same gauge-fixed form in the gauge (43).

The two alternative expansions are useful because the excitations $\mathcal{E}_{\mu}$ and $\mathcal{E}_{\mu}^{\prime}$ are invariant under infinitesimal local particle Lorentz transformations. In these variables, the local Lorentz symmetry is hidden and only the diffeomorphism symmetry is manifest. The Lorentz NG modes lie in the $b_{\mu}$-tranverse components of $\mathcal{E}_{\mu}$ or $\mathcal{E}_{\mu}^{\prime}$. The massive mode $\beta$ joins the diffeomorphism mode in the longitudinal component of $\mathcal{E}_{\mu}$. It can be identified from Eq. (62) as the combination

$$
\beta=\frac{b^{\mu}\left(\mathcal{E}_{\mu}-\frac{1}{2} h_{\mu \nu} b^{\nu}\right)}{b^{\alpha} \hat{b}_{\alpha}}=\mp \hat{b}^{\mu}\left(\mathcal{E}_{\mu}-\frac{1}{2} h_{\mu \nu} b^{\nu}\right) .
$$

Notice that $\beta$ appears here as a diffeomorphism-invariant combination of field components that transform nontrivially.

The choice of diffeomorphism gauge has interesting consequences for $\mathcal{E}_{\mu}$ and $\mathcal{E}_{\mu}^{\prime}$. First, note that $\mathcal{E}_{\mu}$ cannot be gauged to zero, since only 1 degree of freedom $\xi_{\alpha} b^{\alpha}$ appears in its transformation law. However, it is possible to gauge $\mathcal{E}_{\mu}^{\prime}$ to zero. The corresponding gauge condition for $\mathcal{E}_{\mu}$ is $\mathcal{E}_{\mu}=h_{\mu \nu} b^{\nu}$. In either of these gauges, the massive mode becomes

$$
\beta=\mp \frac{1}{2} \hat{b}^{\mu} h_{\mu \nu} \hat{b}^{\nu},
$$

thereby becoming part of the metric. The bumblebee field strength reduces to

$$
B_{\mu \nu}=\left(\partial_{\mu} h_{\nu \sigma}-\partial_{\nu} h_{\mu \sigma}\right) b^{\sigma},
$$

and so is also given by the metric. Evidently, in these gauges the theory is defined entirely in terms of the metric excitations $h_{\mu \nu}$. Requiring $\mathcal{E}_{\mu}$ to vanish or dropping the term $h_{\mu \nu} b^{\nu}$ in Eq. (65) therefore improperly sets to zero the bumblebee field strength $B_{\mu \nu}$, which alters the equations of motion governing the Lorentz NG and massive modes [48]. It follows from Eq. (70) that the effective Lagrange density can contain additional kinetic terms for $h_{\mu \nu}$, beyond those arising from the gravitational action, that originate from the bumblebee kinetic terms $\mathcal{L}_{\mathrm{K}}$. These additional terms propagate the Lorentz NG modes, disguised as massless modes contained in the metric.

\section{EXAMPLES}

In this section, some simple examples are developed to illustrate and enrich the general results obtained in the discussions above. We choose to work within the class of KS bumblebee models with Lagrange density given by Eq. (26), which avoids a priori propagating ghost fields and the complications of nonminimal gravitational couplings. For definiteness, we set $\Lambda=0$ and choose the matter-bumblebee coupling to be

$$
\mathcal{L}_{\mathrm{M}}=-e B_{\mu} J_{\mathrm{M}}^{\mu},
$$

where $J_{\mathrm{M}}^{\mu}$ is understood to be a current formed from matter fields in the theory. In principle, this current could be formed from dynamical fields in the theory or could be prescribed externally. For the latter case, diffeomorphism invariance is explicitly broken unless the current satisfies a suitable differential constraint.

The section begins by presenting some general results for this class of models, including ones related to the equations of motion, conservation laws, and the connection to Einstein-Maxwell electrodynamics. We then turn to a more detailed discussion of three specific cases with differ- 
ent explicit potentials $V(X)$, where the bumblebee field combination $X$ is defined as in Eq. (22). The three cases involve the smooth quadratic potential $V_{S}(X)$ of Eq. (5), the linear Lagrange-multiplier potential $V_{L}(\lambda, X)$ of Eq. (6), and the quadratic Lagrange-multiplier potential $V_{Q}(\lambda, X)$ of Eq. (7). The massive modes are studied for each potential, and their effects on gravity and electromagnetism are explored.

\section{A. General considerations}

\section{Equations of motion and conservation laws}

Varying the Lagrange density (26) with respect to the metric yields the gravitational equations of motion

$$
G^{\mu \nu}=8 \pi G T^{\mu \nu} .
$$

In this expression, the total energy-momentum tensor $T^{\mu \nu}$ can be written as a sum of two terms,

$$
T^{\mu \nu}=T_{\mathrm{M}}^{\mu \nu}+T_{B}^{\mu \nu}
$$

The first is the energy-momentum tensor $T_{\mathrm{M}}^{\mu \nu}$ arising from the matter sector. The second is the energy-momentum tensor $T_{B}^{\mu \nu}$ arising from the bumblebee kinetic and potential terms,

$$
T_{B}^{\mu \nu}=T_{\mathrm{K}}^{\mu \nu}+T_{V}^{\mu \nu}
$$

where $T_{\mathrm{K}}^{\mu \nu}$ and $T_{V}^{\mu \nu}$ are given by

$$
T_{\mathrm{K}}^{\mu \nu}=B^{\mu \alpha} B_{\alpha}^{\nu}-\frac{1}{4} g^{\mu \nu} B_{\alpha \beta} B^{\alpha \beta}
$$

and

$$
T_{V}^{\mu \nu}=-V g^{\mu \nu}+2 V^{\prime} B^{\mu} B^{\nu} .
$$

Varying instead with respect to the bumblebee field generates the remaining equations of motion,

$$
D_{\nu} B^{\mu \nu}=J^{\mu} \text {. }
$$

Like the total energy-momentum tensor, the total current $J^{\mu}$ can be written as the sum of two terms

$$
J^{\mu}=J_{\mathrm{M}}^{\mu}+J_{B}^{\mu} .
$$

The partial current $J_{\mathrm{M}}^{\mu}$ is associated with the matter sector and acts as an external source for the bumblebee field. The partial current $J_{B}^{\mu}$ arises from the bumblebee selfinteraction, and it is given explicitly as

$$
J_{B}^{\mu}=-2 V^{\prime} B^{\mu} .
$$

The contracted Bianchi identities for $G_{\mu \nu}$ lead to conservation of the total energy-momentum tensor,

$$
D_{\mu} T^{\mu \nu} \equiv D_{\mu}\left(T_{\mathrm{M}}^{\mu \nu}+T_{B}^{\mu \nu}\right)=0 .
$$

The antisymmetry of the bumblebee field strength $B_{\mu \nu}$ implies the remnant constraint (25) and hence a second conservation law,

$$
D_{\mu} J^{\mu} \equiv D_{\mu}\left(J_{\mathrm{M}}^{\mu}+J_{B}^{\mu}\right)=0 .
$$

Note that this second conservation law is a special feature of KS bumblebee models. It is a direct consequence of choosing the a priori ghost-free action (26). Note also that this conservation law holds even though the potential term $V$ excludes any local $\mathrm{U}(1)$ gauge symmetry in these models.

\section{Bumblebee currents}

The bumblebee current $J_{B}^{\mu}$ defined in Eq. (79) vanishes when $V^{\prime}=0$. This situation holds for the vacuum solution and for NG modes, and it then follows from Eq. (81) that the matter current $J_{\mathrm{M}}^{\mu}$ is covariantly conserved. However, $V^{\prime} \neq 0$ in the presence of a nonzero massive mode or a nonzero linear Lagrange multiplier, whereupon the bumblebee current $J_{B}^{\mu}$ acts as an additional source for the bumblebee field equation (77). A nonzero massive mode or nonzero linear Lagrange multiplier contributes to the bumblebee component $T_{B}^{\mu \nu}$ of the energy-momentum tensor too, and it therefore also acts as an additional source for the gravitational field equations (72).

The contributions to the energy-momentum tensor $T_{B}^{\mu \nu}$ stemming from $V^{\prime} \neq 0$ can be positive or negative. This is a generic feature, as can be seen from the expression for $T_{V}^{\mu \nu}$ in Eq. (17) and the general bumblebee Lagrange density (19). The full energy-momentum tensor $T^{\mu \nu}$ is conserved, but the prospect of negative values for $T_{B}^{\mu \nu}$ implies stability issues for the models. Under suitable circumstances, for example, unbounded negative values of $T_{B}^{\mu \nu}$ can act as unlimited sources of energy for the matter sector. Stability is also a potential issue in the absence of matter. For example, for the case where $J_{\mathrm{M}}^{\mu}$ is disregarded and the potential involves a linear Lagrange multiplier producing $\lambda \neq 0$ on shell, at least one set of initial values is known that yields a negative-energy solution [54].

Whether instabilities occur in practice depends on the form of the Lagrange density and on the initial conditions. The situation is comparatively favorable for KS bumblebee models because the extra conservation law (81) can play a role. In principle, negative-energy contributions in the initial state can be eliminated by choosing initial conditions such that $V^{\prime}=0$, while the conservation law can prevent the eventual development of a destabilizing mode. One way to see this is to expand the conservation law Eq. (81) at leading order in a Minkowski background. For nonzero $\left\langle B_{0}\right\rangle$, we obtain

$$
\partial_{0} V^{\prime} \approx \frac{1}{2 B^{0}}\left(\partial_{\mu} J_{\mathrm{M}}^{\mu}-2 V^{\prime} \partial_{\mu} B^{\mu}-2 B^{j} \partial_{j} V^{\prime}\right) .
$$

Suppose the matter current is independently conserved, $D_{\mu} J_{\mathrm{M}}^{\mu} \approx \partial_{\mu} J_{\mathrm{M}}^{\mu}=0$. The initial condition $V^{\prime}=0$ then implies $\partial_{0} V^{\prime}=0$ initially. Taking further derivatives shows that all time derivatives of $V^{\prime}$ vanish initially and so $V^{\prime}$ remains zero for all time, indicating stability is maintained. 
It is interesting to note that the matter-current conservation law $D^{\mu} J_{\mu}^{\mathrm{M}}=0$ emerges naturally in the limit of vanishing NG and massive modes, where the bumblebee field reduces to its vacuum value $B_{\mu} \rightarrow\left\langle B_{\mu}\right\rangle=b_{\mu}$. This agrees with a general conjecture made in Ref. [4]. The line of reasoning is as follows. In the limit, the bumblebee theory with spontaneous Lorentz violation takes the form of a theory with explicit Lorentz violation with couplings only to $b_{\mu}$. However, theories with explicit Lorentz violation contain an incompatibility between the Bianchi identities and the energy-momentum conservation law. The incompatibility is avoided for spontaneous Lorentz violation by the vanishing of a particular variation in the action, which reduces in the limit to the requirement of current conservation $D^{\mu} J_{\mu}^{\mathrm{M}}=0$. Nonetheless, if $B_{\mu}$ is excited away from this limit, current conservation in the matter sector may fail to hold.

In practice, the potential instability may be irrelevant for physics. For example, if a bumblebee model is viewed as an effective field theory emerging from an underlying stable quantum theory of gravity, any apparent instabilities may reflect incomplete knowledge of constraints on the massive modes or of countering effects that come into play at energy scales above those of the effective theory. Under these circumstances, in KS bumblebee models it may suffice for practical purposes simply to postulate that the matter and bumblebee currents do not mix and hence obey separate conservation laws,

$$
D_{\mu} J_{M}^{\mu}=0, \quad D_{\mu} J_{B}^{\mu}=0 .
$$

For instance, one can impose that only matter terms $\mathcal{L}_{M}$ with a global U(1) symmetry are allowed, as is done for the case of Minkowski spacetime in Ref. [50]. Another option might be to disregard $J_{\mu}^{\mathrm{M}}$ altogether and allow couplings between the matter and bumblebee fields only through gravity. In what follows, we adopt Eqs. (83) as needed to investigate modifications of gravity arising from massive modes and to study the conditions under which EinsteinMaxwell solutions emerge in bumblebee models.

\section{Bumblebee electrodynamics}

An interesting aspect of the KS bumblebee models is their prospective interpretation as alternative theories of electrodynamics with physical signatures of Lorentz violation [5]. In this approach, basic electrodynamics properties such as the masslessness of photons are viewed as consequences of spontaneous local Lorentz and diffeomorphism breaking rather than of exact local U(1) symmetry. A key point is that KS bumblebee models have no dependence on second time derivatives of $B_{0}$. They therefore feature an additional primary constraint relative to the bumblebee models (19) with more general kinetic terms. The primary constraint generates a secondary constraint in the form of a modified Gauss law, which permits a physical interpretation of the theory in parallel to electrodynamics.
This modified Gauss law is unavailable to other bumblebee models.

In a suitable limit, the equations of motion (72) and (77) for the KS bumblebee models reduce to the EinsteinMaxwell equations in a fixed gauge for the metric and photon fields. To demonstrate this, we work in asymptotically Minkowski spacetime and choose the gauge in which the bumblebee field is given by Eq. (59). The limit of interest is $\beta \rightarrow 0$, corresponding to zero massive mode. For the case with the Lagrange-multiplier potential (6), where $\beta$ is constrained to zero, an equivalent result also follows in the limit $\lambda \rightarrow 0$. Since $T_{V}^{\mu \nu}$ acquires nonzero contributions only from the massive mode or linear Lagrange multiplier, it follows that as $\beta \rightarrow 0$ or $\lambda \rightarrow 0$ the bumblebee energy-momentum tensor $T_{B}^{\mu \nu}$ reduces to that of Einstein-Maxwell electrodynamics,

$$
T_{B}^{\mu \nu} \rightarrow T_{\mathrm{K}}^{\mu \nu} \equiv T_{\mathrm{EM}}^{\mu \nu}=F^{\mu \alpha} F_{\alpha}{ }^{\nu}-\frac{1}{4} g_{\mu \nu} F_{\alpha \beta} F^{\alpha \beta} .
$$

In this equation, the bumblebee field strength is reinterpreted as the electromagnetic field strength

$$
B_{\mu \nu} \equiv F_{\mu \nu}=\partial_{\mu} A_{\nu}-\partial_{\nu} A_{\mu},
$$

via the mode expansion (59), and the gravitational field equations (72) reduce to their Einstein-Maxwell counterparts. Similarly, the bumblebee current $J_{B}^{\mu}$ in Eq. (79) vanishes as $\beta \rightarrow 0$ or $\lambda \rightarrow 0$ because $V^{\prime} \rightarrow 0$. This leaves only the conventional matter current $J_{\mathrm{M}}^{\mu}$, which by virtue of Eq. (81) obeys covariant conservation. The bumblebee field equations (77) therefore also reduce to their Einstein-Maxwell counterparts.

Even when $\beta$ vanishes, the interpretation of the model as bumblebee electrodynamics can in principle be distinguished from conventional Einstein-Maxwell electrodynamics through nontrivial SME-type couplings involving the vacuum value $b_{\mu}$ in the matter equations of motion. Depending on the types of couplings appearing in the matter-sector Lagrange density $\mathcal{L}_{\mathrm{M}}$, a variety of effects could arise. At the level of the minimal SME, for example, axial vector couplings involving $b_{\mu}$ can be sought in numerous experiments, including ones with electrons $[22,23]$, protons and neutrons [25,26], and muons [29]. Other types of SME coefficients can also be generated from the vacuum value $b_{\mu}$. For example, the nonzero symmetric traceless SME coefficients $c_{\mu \nu}$ might emerge via the symmetric traceless product $b_{\mu} b_{\nu}-b^{\alpha} b_{\alpha} \eta_{\mu \nu} / 4$, and experimental searches for these include ones with electrons [22,24], protons, and neutrons [25,26].

If the model conditions permit the field $\beta$ to be nonzero, further deviations from Einstein-Maxwell electrodynamics can arise. For example, in the weak-field regime where $\beta \hat{b}_{\mu}$ is small compared to $b_{\mu}$, the bumblebee current $J_{B}^{\mu}$ is linear in $\beta$ and acts as an additional source in the equation of motion (77) for the field strength $B_{\mu \nu}$. Effects of this type are investigated in the next few subsections. For large 
fields, nonlinear effects in the bumblebee current and energy-momentum tensor may also play a role. However, the field value of $\beta$ is typically suppressed when the mass of $\beta$ is large, so if the mass is set by the Planck scale then the linear approximation is likely to suffice for most realistic applications.

In the limit of zero $\beta$, the physical fields are the field strengths $B_{\mu \nu}$ and the gravitational field. Excitations of the bumblebee field $B_{\mu}$ in the classical theory are then unobservable, in parallel with the potential $A_{\mu}$ in classical electromagnetism. This may have consequences for leading-order effects in the weak-field limit. For example, the weak static limit in general bumblebee models (19) requires $B_{\mu}$ itself to be time independent. However, at leading order in the KS bumblebee model, it can suffice to require that only the field strength $B_{\mu \nu}$ be time independent while $B_{\mu}$ itself has time dependence, in analogy with Maxwell electrodynamics in Minkowski spacetime. For example, the Coulomb electric field $\vec{E}=-\partial_{0} \vec{A}$ for a static point charge $q$ emerges in temporal gauge $A_{0}=0$ from a time-dependent $A_{\mu}=\left(0, t \partial_{j} \Phi_{q}\right)$, where $\Phi_{q}$ is the Coulomb potential. Likewise, in certain leading-order weak static solutions for which $\beta$ acts as a source of charge, the bumblebee field $B_{\mu}$ may naturally exhibit potential lines converging at the source of charge, similar to the physical configurations with singular derivatives of $A_{\mu}$ that occur in classical electrodynamics.

Another interesting issue is the role of quantum effects. Since bumblebee electrodynamics involves gravity, it faces the same quantization challenges as other theories of gravity and electrodynamics, including Einstein-Maxwell theory. Some work on the renormalization structure at one loop has been performed [44,55], and in the limit of zero massive mode and in Minkowski spacetime the usual properties of quantum electrodynamics are expected to hold. Addressing this issue in detail is an interesting open problem that lies beyond the scope of this work.

\section{B. Smooth quadratic potential}

This subsection considers the specific KS bumblebee model (26) having smooth quadratic potential (5) with the definition (22):

$$
V=V_{S}(X)=\frac{1}{2} \kappa\left(B_{\mu} g^{\mu \nu} B_{\nu} \pm b^{2}\right)^{2} .
$$

For definiteness, we adopt the mode expansion $B_{\mu}=$ $b_{\mu}+\mathcal{E}_{\mu}$ of Eq. (60) in a Minkowski background and assume weak fields $h_{\mu \nu}$ and $\mathcal{E}_{\mu}$ so that the gravitational and bumblebee equations of motion (72) and (77) can be linearized.

One goal is to investigate deviations from EinsteinMaxwell theory arising from the presence of a weak nonzero massive mode $\beta$. We therefore focus on dominant corrections to the linearized Einstein-Maxwell equations arising from $\beta$. In this approximation, the bumblebee component of the energy-momentum tensor becomes

$$
T_{B}^{\mu \nu} \approx T_{\mathrm{EM}}^{\mu \nu}+4 \kappa\left(b^{\alpha} \hat{b}_{\alpha}\right) b^{\mu} b^{\nu} \beta,
$$

where $T_{\mathrm{EM}}^{\mu \nu}$ is the zero- $\beta$ limit of $T_{\mathrm{K}}^{\mu \nu}$ given in Eq. (84) and the other term arises from $T_{V}^{\mu \nu}$. The bumblebee current reduces to

$$
J_{B}^{\mu} \approx-4 \kappa\left(b^{\alpha} \hat{b}_{\alpha}\right) b^{\mu} \beta .
$$

The constraint obtained by assuming current conservation in the matter sector becomes

$$
b^{\mu} \partial_{\mu} \beta \approx 0 .
$$

These expressions reveal that at leading order the massive mode $\beta$ acts as a source for gravitation and electrodynamics, subject to the constraint (89).

Note that the linearization procedure can alter the time behavior and dynamics of the fields in the presence of a nonzero massive mode $\beta$. Suppose, for example, that $b_{\mu}$ is timelike and we adopt the global observer frame in which $b_{\mu}=(b, 0,0,0)$. Nonlinearities in the current $J_{B}^{\mu}$ associated with nonzero $\beta$ generate time dependence for most solutions because the spatial current $\vec{J}_{B}$ does work on the field strength $B_{\mu \nu}$. However, the linearization (88) implies $J_{B}^{\mu}$ is nonzero only along the direction of $b^{\mu}$, reducing to a pure charge density $J_{B}^{\mu} \approx\left(\rho_{B}, 0,0,0\right)$ with $\rho_{B} \approx$ $-4 \kappa b^{2} \beta$, and the current-conservation law (89) then requires $\rho_{B}$ and hence $\beta$ to be static. Nonetheless, the linearization procedure captures the dominant effects of nonzero $\beta$.

\section{Propagating modes}

To study free propagation of the gravitational and bumblebee fields in the absence of charge and matter, set $J_{\mathrm{M}}^{\mu}=$ $T_{\mathrm{M}}^{\mu \nu}=0$ in the linearized equations of motion. The gravitational equations (72) then become

$$
\begin{aligned}
& \square \bar{h}_{\mu \nu}+\eta_{\mu \nu} \partial^{\alpha} \partial^{\beta} \bar{h}_{\alpha \beta}-\partial^{\alpha} \partial_{\mu} \bar{h}_{\alpha \nu}-\partial^{\alpha} \partial_{\nu} \bar{h}_{\alpha \mu} \\
& \quad \approx-64 \pi G \kappa\left(b^{\alpha} \hat{b}_{\alpha}\right) b_{\mu} b_{\nu} \beta .
\end{aligned}
$$

Contributions from $T_{\mathrm{EM}}^{\mu \nu}$ are second-order in $\mathcal{E}_{\mu}$ and can be neglected in this context. The bumblebee equations (77) reduce to

$$
\square \mathcal{E}_{\mu}-\partial_{\mu} \partial^{\nu} \mathcal{E}_{\nu} \approx 4 \kappa\left(b^{\alpha} \hat{b}_{\alpha}\right) b_{\mu} \beta .
$$

Note that the massive mode $\beta$ is defined by Eq. (68) in terms of $\mathcal{E}_{\mu}$ and $h_{\mu \nu}$, so it is not an independent field in these equations. Note also that the linearized currentconservation law (89) follows by taking a derivative of Eq. (91). 
To investigate the behavior of the massive mode $\beta$, it is convenient to combine the above two equations to obtain

$$
\begin{gathered}
\square \beta-4 \kappa\left(b^{\alpha} b_{\alpha}\right)\left(1+4 \pi G b^{\beta} b_{\beta}\right) \beta \\
\approx \frac{1}{b^{\gamma} \hat{b}_{\gamma}} b^{\lambda} \partial_{\lambda} \partial^{\mu}\left(\mathcal{E}_{\mu}-\bar{h}_{\mu \nu} b^{\nu}\right) .
\end{gathered}
$$

At first glance, this equation might suggest that $\beta$ can be a propagating massive field. However, $\beta$ depends on the fields appearing on the right-hand side, so the modes are still coupled and care is required in determining the dispersion properties, including the mass value.

A suitable choice of diffeomorphism gauge clarifies matters. The modes remain entangled in the harmonic gauge (42) and also in the barred axial gravitational gauge $\bar{h}_{\mu \nu} b^{\nu}=0$. However, a sufficient decoupling can be achieved by adopting the axial gravitational gauge (43) and by decomposing $\mathcal{E}_{\mu}$ into pieces parallel and perpendicular to $b_{\mu}$. In this gauge, the longitudinal component $\mathcal{E}$ of $\mathcal{E}_{\mu}$ reduces to $\mathcal{E}=\beta$, so that

$$
\mathcal{E}_{\mu}=\mathcal{E}_{\mu}^{t}+\mathcal{E} \hat{b}_{\mu}=A_{\mu}+\beta \hat{b}_{\mu}
$$

In this equation, we write $\mathcal{E}_{\mu}^{t} \equiv A_{\mu}$ for the transverse components of $\mathcal{E}_{\mu}$ to make easier the task of tracking $\beta$-dependent deviations from conventional electrodynamics. These components satisfy the axial condition $A_{\mu} b^{\mu}=$ 0 . We emphasize that the axial condition is not a gauge choice. It is a consequence of projecting along $b_{\mu}$ and is independent of the gravitational gauge fixing. It does, however, have the same mathematical form as an axialgauge condition in electrodynamics, even though the bumblebee models have no U(1) symmetry.

With these choices, the usual Einstein equations for $\bar{h}_{\mu \nu}$ in axial gravitational gauge are recovered from the result (90) when $\beta$ vanishes [5]. For nonzero $\beta$, only one linearly independent combination of the Einstein equations is changed. It can be written

$$
\square \bar{h}+2 \partial^{\mu} \partial^{\nu} \bar{h}_{\mu \nu} \approx-64 \pi G \kappa\left(b^{\alpha} \hat{b}_{\alpha}\right)\left(b^{\beta} b_{\beta}\right) \beta .
$$

Since the usual propagation-transverse components of $\bar{h}_{\mu \nu}$ remain unaffected, the physical graviton modes propagate normally. The bumblebee equations (91) become

$$
\square A_{\mu}-\partial_{\mu} \partial^{\nu} A_{\nu}+\frac{1}{b^{\beta} b_{\beta}} b_{\mu} b^{\alpha} \partial_{\alpha} \partial^{\nu} A_{\nu} \approx 0
$$

and

$$
\square \beta-4 \kappa\left(b^{\alpha} b_{\alpha}\right) \beta \approx \frac{1}{b^{\beta} \hat{b}_{\beta}} b^{\alpha} \partial_{\alpha} \partial^{\mu} A_{\mu}
$$

When the massive mode $\beta$ vanishes, these equations re- duce to those of electrodynamics in axial gauge [5]. A nonzero value of $\beta$ modifies the equations, but the usual propagation-transverse components of $A_{\mu}$ and hence the physical photon modes remain unaffected.

Since $\beta$ and $A_{\mu}$ are independent fields, the dispersion properties of the massive mode $\beta$ can be identified from Eq. (96). Any solutions of the theory describing freely propagating modes are required to obey the boundary conditions that the spacetime be asymptotically flat and that the bumblebee fields vanish at infinity. At linear order, these solutions are formed from harmonic plane waves with energy-momentum vectors $p^{\mu}=(E, \vec{p})$ obeying suitable dispersion relations. The modes satisfying the asymptotic boundary conditions are then constructed as Fourier superpositions of the plane-wave solutions. For the massive mode $\beta$, the constraint equation (89) imposes the additional requirement

$$
b^{\mu} p_{\mu} \approx 0 .
$$

Any freely propagating massive mode is therefore constrained to have an energy-momentum vector orthogonal to the vacuum value $b_{\mu}$. For harmonic-mode solutions to the equations of motion, this requirement implies that the term on the right-hand side of Eq. (96) vanishes in Fourier space. The resulting dispersion law for the massive mode $\beta$ is then

$$
p^{\mu} p_{\mu} \approx-4 \kappa b^{\alpha} b_{\alpha}
$$

which involves the squared-mass parameter

$$
M_{\beta}^{2}=4 \kappa b^{\alpha} b_{\alpha} .
$$

The sign of $M_{\beta}^{2}$ depends on whether $b_{\mu}$ is timelike or spacelike. The scale of $M_{\beta}^{2}$ depends on both $\kappa$ and $b$. Inspection of Eqs. (94) or (96) shows that the limits $\left|M_{\beta}\right|^{2} \rightarrow \infty$ or $\kappa \rightarrow \infty$ with $b_{\mu}$ fixed are equivalent to taking the limit of vanishing massive mode, $\beta \rightarrow 0$. The discussion in Sec. IVA 3 then implies that the limit of large $\left|M_{\beta}\right|^{2}$ approximates Einstein-Maxwell theory.

For the case of a timelike vacuum value $b_{\mu}$, the parameter $M_{\beta}^{2}=-4 \kappa b^{2}$ has the wrong sign for a physical mass. Adopting the global observer frame in which $b_{\mu}=$ $(b, 0,0,0)$, we see that the constraint (97) forces the energy $E$ of this mode to vanish, leaving the condition that the magnitude of the momentum must remain fixed at $|\vec{p}|=$ $\left|M_{\beta}\right|$. A time-independent mode with fixed spatial wavelength cannot be Fourier superposed to form a physical wave packet satisfying the asymptotic boundary conditions. It follows that no physical propagating massive mode appears when $b_{\mu}$ is timelike.

If instead the vacuum value $b_{\mu}$ is spacelike, then $M_{\beta}^{2}=$ $4 \kappa b^{2}$ is positive and is a candidate for a physical mass. Choose the global observer coordinate system such that the 
spacelike $b_{\mu}$ takes the form $b_{\mu}=(0,0,0, b)$. The constraint (97) then requires the vanishing of the momentum along the spatial direction $b_{3}$, and the dispersion law (98) becomes

$$
E^{2}-\left(p_{x}^{2}+p_{y}^{2}\right) \approx M_{\beta}^{2} .
$$

This suggests that $\beta$ could propagate as a harmonic plane wave along spatial directions transverse to $b_{\mu}$. However, these harmonic plane waves are constant in $z$ and hence fail to satisfy the asymptotic boundary conditions as $z \rightarrow \pm \infty$ unless their amplitude is zero. Since all harmonics propagate under the same constraint (97), no Fourier superposition can be constructed to obey the boundary conditions. It follows that no physical propagating massive mode exists for the case of a spacelike vector either.

The above results can also be confirmed by obtaining the eigenvalues of the Fourier-transformed equations of motion, which form a $14 \times 14$ matrix determining the nature of the modes. Consider, for example, the case of purely timelike $b_{\mu}$. In the axial gravitational gauge, four of the modes are zero, corresponding to the four fixed degrees of freedom. Another four eigenvalues describe modes propagating via a massless dispersion law, corresponding to the two photon and two graviton modes. One eigenvalue has zero energy and sets $|\vec{p}|=\left|M_{\beta}\right|$, corresponding to the massive mode $\beta$. The remaining 5 modes are auxiliary, and all have zero energy. Related results can be obtained for the case of spacelike $b_{\mu}$.

\section{Weak static limit}

Although the massive mode $\beta$ is nonpropagating, it plays the role of an auxiliary field and can thereby produce various effects. For example, its presence can affect the weak static limits of the gravitational and bumblebee interactions. In particular, a nonzero $\beta$ can modify the forms of both the Newton and Coulomb potentials.

To demonstrate this, we consider the weak static limit of the field equations with an external matter sector containing massive point charges. The matter Lagrange density can be taken as

$$
\begin{aligned}
\mathcal{L}_{\mathrm{M}}= & \sum_{n} m_{n} \int d \tau\left[g_{\mu \nu} \frac{d x_{n}^{\mu}}{d \tau} \frac{d x_{n}^{\nu}}{d \tau}\right]^{1 / 2} \delta^{4}\left(x-x_{n}(\tau)\right) \\
& +\sum_{n} q_{n} \int d \tau B_{\mu}(x) \frac{d x_{n}^{\mu}}{d \tau} \delta^{4}\left(x-x_{n}(\tau)\right)
\end{aligned}
$$

where $m_{n}$ and $q_{n}$ are the masses and charges of the point particles, respectively. The energy-momentum tensor for the matter sector is

$$
T_{\mathrm{M}}^{\mu \nu}=\frac{1}{e} \sum_{n} m_{n} \int d \tau \frac{d x_{n}^{\mu}}{d \tau} \frac{d x_{n}^{\nu}}{d \tau} \delta^{4}\left(x-x_{n}(\tau)\right)
$$

Its combination with $T_{B}^{\mu \nu}$ given in Eq. (74) forms the total energy-momentum tensor, which is conserved. The mattersector current $J_{\mathrm{M}}^{\mu} \equiv\left(\rho_{q}, \vec{J}\right)$ is

$$
J_{\mathrm{M}}^{\mu}=\frac{1}{e} \sum_{n} q_{n} \int d \tau \frac{d x_{n}^{\mu}}{d \tau} \delta^{4}\left(x-x_{n}(\tau)\right) .
$$

It is conserved, $D_{\mu} J_{\mathrm{M}}^{\mu}=0$.

The standard route to adopting the weak static limit is to linearize the equations of motion in the fields and then discard all time derivatives. This meets no difficulty under suitable circumstances, such as the vacuum value $b_{\mu}$ being purely spacelike, and we follow this route in the present subsection. However, in certain cases additional care is required because the $b_{\mu}$-transverse components of the bumblebee field obey the axial condition $b^{\mu} \mathcal{E}_{\mu}^{t}=0$, which can imply time dependence even for static fields. For example, as discussed in Sec. IVA 3, the case of purely timelike $b_{\mu}$ and zero $\beta$ is equivalent to electrodynamics in temporal gauge, for which the static Coulomb potential involves a linear time dependence in $\vec{A}$. The corresponding weak static limit with nonzero $\beta$ can therefore be expected to exhibit this feature. We return to this issue in the next subsection.

To proceed, it is useful to choose a diffeomorphism gauge that simplifies the Einstein tensor and allows direct comparison between the geodesic equation and the Newton force law. One possible choice is the harmonic gauge (42), in which the static gravitational potential $\Phi_{g}$ is related to the metric by $\Phi_{g}=-\frac{1}{2} h_{00}=-\frac{1}{2} \bar{h}_{00}+\frac{1}{4} \bar{h}$. The linearized gravitational equations become

$$
-\frac{1}{2} \square \bar{h}^{\mu \nu} \approx 8 \pi G\left[T_{\mathrm{M}}^{\mu \nu}+T_{\mathrm{EM}}^{\mu \nu}+4 \kappa\left(b^{\alpha} \hat{b}_{\alpha}\right) b^{\mu} b^{\nu} \beta\right]
$$

where $\beta$ is the massive mode given in terms of $\mathcal{E}_{\mu}$ and $h_{\mu \nu}$ in Eq. (68), and $T_{\mathrm{EM}}^{\mu \nu}$ is the zero- $\beta$ form of $T_{\mathrm{K}}^{\mu \nu}$ given in Eq. (84).

We can now extract the relevant equations in the weak static limit. The equation for the gravitational potential $\Phi_{g}$ is the 00 component of the trace-reversed form of Eq. (104),

$$
\nabla^{2} \Phi_{g} \approx 8 \pi G\left[\bar{T}_{\mathrm{M}}^{00}+\bar{T}_{\mathrm{EM}}^{00}+2 \kappa\left(b^{\alpha} \hat{b}_{\alpha}\right)\left(b_{0}^{2}+\vec{b}^{2}\right) \beta\right]
$$

where the trace-reversed energy-momentum tensors are defined by $\bar{T}_{\mu \nu} \equiv T_{\mu \nu}-\eta_{\mu \nu} T_{\alpha}^{\alpha}$ and are evaluated in the static limit. The weak static bumblebee equations become 


$$
\begin{aligned}
\vec{\nabla}^{2} \mathcal{E}_{0} & \approx \rho_{q}+4 \kappa\left(b^{\alpha} \hat{b}_{\alpha}\right) b_{0} \beta, \\
\vec{\nabla}^{2} \overrightarrow{\mathcal{E}}-\vec{\nabla}(\vec{\nabla} \cdot \overrightarrow{\mathcal{E}}) & \approx \vec{J}+4 \kappa\left(b^{\alpha} \hat{b}_{\alpha}\right) \vec{b} \beta,
\end{aligned}
$$

where $\rho_{q}$ and $\vec{J}$ are static charge and current sources.

The above equations show that the massive mode $\beta$ can be viewed in the weak static limit as a simultaneous source of energy density, charge density, and current density. The relative weighting of the contributions is controlled by the relative sizes of the components of $b_{\mu}$. In fact, the same is also true of contributions to gravitomagnetic and higherorder gravitational effects arising from other components of the gravitational equations. In the interpretation as bumblebee electrodynamics, the equations show that we can expect deviations from the usual Newton and Coulomb force laws when $\beta$ is nonzero. Since the effects are controlled by components of $b_{\mu}$, they are perceived differently from different observer frames, much like charge and current in ordinary electrodynamics. Also, the unconventional source terms are linear in $\beta$, so the solutions can be interpreted as superpositions of conventional gravitational and electrodynamic fields with effects due to $\beta$. However, since $\beta$ itself is formed as the combination (68) of the metric and bumblebee fields, the extra source terms in fact reflect contributions from the gravitational and electrodynamic fields that are absent in the Einstein-Maxwell limit.

\section{Timelike case}

In this subsection, we consider in more detail the weak static limit with timelike $b_{\mu}$. We solve explicitly the weak static equations in the special observer frame for which $b_{\mu}=(b, 0,0,0)$ is purely timelike, and we provide some remarks about the more general case. The special frame might be identified with the natural frame of the cosmic microwave background. However, in laboratory searches for modifications to weak static gravitation and electrodynamics, this frame can at best be an approximation because the Earth rotates and revolves about the Sun, which in turn is moving with respect to the cosmic microwave background. Nonetheless, the explicit solutions obtained here offer useful insight into the modifications to the Newton and Coulomb potentials that are introduced by the massive mode.

For the purely timelike case, both $h_{\mu \nu}$ and $B_{\mu \nu}$ are expected to be time independent in the weak static limit. However, time dependence must appear in $\overrightarrow{\mathcal{E}}$ even in the static limit because as $\beta \rightarrow 0$ the match to electrodynamics arises in the temporal gauge, for which the standard Coulomb solution has a linear time dependence. For this reason, in taking the weak static limit in what follows, we keep time derivatives acting on the vector components $\overrightarrow{\mathcal{E}}$.

The analysis for purely timelike $b_{\mu}$ could be performed in the harmonic gauge, but it turns out that choosing the transverse gauge instead results in the complete decoupling from the massive mode of nine of the 10 gravitational equations. The transverse gauge is defined by

$$
\partial^{j} h_{0 j}=0, \quad \partial^{j} h_{j k}=\frac{1}{3} \partial_{k} h_{j}^{j},
$$

where $j, k$ range over the spatial directions. In this gauge, $G_{00} \approx-\nabla^{2} h_{00}=2 \nabla^{2} \Phi_{g}$, where $\Phi_{g}$ is the gravitational potential.

Taking the weak static limit of the gravitational and bumblebee equations of motion in this gauge yields

$$
\begin{aligned}
\nabla^{2} \Phi_{g} & \approx 4 \pi G\left(T_{\mathrm{M}}^{00}+T_{\mathrm{EM}}^{00}-4 \kappa b^{3} \beta\right), \\
\vec{\nabla}^{2} \mathcal{E}_{0}-\vec{\nabla} \cdot \partial_{0} \overrightarrow{\mathcal{E}} & \approx \rho_{q}-4 \kappa b^{2} \beta, \\
\square \overrightarrow{\mathcal{E}}-\vec{\nabla}(\vec{\nabla} \cdot \overrightarrow{\mathcal{E}}) & \approx \vec{J} .
\end{aligned}
$$

The other nine components of $h_{\mu \nu}$ obey equations that are identical to those of general relativity in the weak static limit. In Eq. (108), $T_{\mathrm{M}}^{00}$ is the static energy density in the matter sector, and $T_{\mathrm{EM}}^{00}$ is the static 00 component of the zero- $\beta$ limit of $T_{\mathrm{K}}^{\mu \nu}$ given in Eq. (84). The charge density $\rho_{q}$ and current density $\vec{J}$ are also understood to be timeindependent distributions.

The explicit form of the massive mode $\beta$ follows from Eq. (68) and is found to be

$$
\beta=\mathcal{E}_{0}-b \Phi_{g} .
$$

For purely timelike $b_{\mu}$, the constraint (89) reduces to

$$
\partial_{0} \beta \approx 0,
$$

so $\beta$ is time independent at this order. As described in the previous subsection, $\beta$ can be interpreted as a source for $\Phi_{g}$ and $\mathcal{E}_{0}$. Here, $\beta$ acts as a static source of energy density and charge density with

$$
\begin{aligned}
T_{B}^{\mu \nu} & =b \rho_{B} \eta^{0 \mu} \eta^{0 \nu}, \\
J_{B}^{\mu} & =\left(\rho_{B}, 0,0,0\right),
\end{aligned}
$$

where $\rho_{B}=-4 \kappa b^{2} \beta$.

To find the explicit modifications to the gravitational and electromagnetic potentials caused by $\beta$, we focus on the case of a point particle of mass $m$ and charge $q$ located at the origin. The source terms in this case have the form

$$
\begin{aligned}
T_{\mathrm{M}}^{00} & =\rho_{m}=m \delta^{(3)}(\vec{x}), \\
J_{\mathrm{M}}^{0} & \equiv \rho_{q}=q \delta^{(3)}(\vec{x}),
\end{aligned}
$$

and all other components of $T_{\mathrm{M}}^{\mu \nu}$ and $J_{\mathrm{M}}^{\mu}$ are zero. In the absence of Lorentz violation, the weak static solution to the Einstein-Maxwell equations with these source terms consists of the linearized Reissner-Nordström metric and corresponding electromagnetic fields. Denote by $\Phi_{m}$ the associated gravitational potential. It obeys 


$$
\vec{\nabla}^{2} \Phi_{m}=4 \pi G\left(\rho_{m}+T_{\mathrm{EM}}^{00}\right) .
$$

In the limit $q \rightarrow 0$, it reduces to the Newton gravitational potential and obeys the usual Poisson equation $\nabla^{2} \Phi_{m}=$ $4 \pi G \rho_{m}$. Similarly, denote the conventional Coulomb potential by $\Phi_{q}$. It obeys the Poisson equation

$$
\nabla^{2} \Phi_{q}=-\rho_{q}
$$

It is also convenient to express the massive mode $\beta$ in terms of a potential $\Phi_{B}$. We define

$$
\beta \equiv \frac{1}{\left|M_{\beta}\right|^{2}} \vec{\nabla}^{2} \Phi_{B}(\vec{x})
$$

where $\left|M_{\beta}\right|^{2}$ is the absolute value of the squared mass in Eq. (99). Note that $\Phi_{B}$ is time independent due to the constraint (110). Since $\beta$ acts as a source of static charge density given by Eq. (111), the definition of $\Phi_{B}$ can be interpreted as a third Poisson equation,

$$
\vec{\nabla}^{2} \Phi_{B}=-\rho_{B} .
$$

The time independence of both $\beta$ and $\Phi_{B}$ means they are determined by their initial values.

For the point particle of mass $m$ and charge $q$, the general solution for the gravitational potential $\Phi_{g}$ and the bumblebee field $\mathcal{E}_{\mu}$ in the weak static limit can be expressed in terms of the conventional potentials $\Phi_{m}$ and $\Phi_{q}$ and the bumblebee potential $\Phi_{B}$. We obtain

$$
\begin{aligned}
& \Phi_{g}=\Phi_{m}-4 \pi G b \Phi_{B}, \\
& \mathcal{E}_{0}=b \Phi_{m}-4 \pi G b^{2} \Phi_{B}+\frac{1}{\left|M_{\beta}\right|^{2}} \vec{\nabla}^{2} \Phi_{B}, \\
& \mathcal{E}_{j}=t \partial_{j}\left[\Phi_{q}+b \Phi_{m}+\left(1-4 \pi G b^{2}\right) \Phi_{B}+\frac{1}{\left|M_{\beta}\right|^{2}} \vec{\nabla}^{2} \Phi_{B}\right] .
\end{aligned}
$$

The corresponding static gravitational field $\vec{g}$, static electric field $\vec{E}$, and static magnetic field $\vec{B}$ are found to be

$$
\begin{aligned}
\vec{g} & =-\vec{\nabla} \Phi_{m}+4 \pi G b \vec{\nabla} \Phi_{B}, \\
\vec{E} & =-\vec{\nabla} \Phi_{q}-\vec{\nabla} \Phi_{B}, \\
\vec{B} & =0 .
\end{aligned}
$$

The static Maxwell equations include a modified Gauss law

$$
\begin{aligned}
\vec{\nabla} \cdot \vec{E} & =-\vec{\nabla}^{2} \Phi_{q}-\vec{\nabla}^{2} \Phi_{B} \\
& =\rho_{q}+\rho_{B},
\end{aligned}
$$

together with the usual static laws

$$
\begin{aligned}
\vec{\nabla} \cdot \vec{B} & =0, \\
\vec{\nabla} \times \vec{E} & =0, \\
\vec{\nabla} \times \vec{B} & =0 .
\end{aligned}
$$

These equations demonstrate for the purely timelike case the ways in which a nonzero massive mode $\beta$ modifies the conventional static gravitational and electrodynamic fields.

Compared to electrodynamics, the purely timelike KS bumblebee model introduces an additional degree of freedom into the problem of determining the static electric field $\vec{E}$. This extra degree of freedom is the massive mode $\beta$, and the extent of its effects depends on the initial conditions for $\beta$. In the absence of a satisfactory underlying theory predicting $\beta$ or of direct experimental observation of $\beta$, these initial conditions are undetermined.

One natural choice is to adopt $\Phi_{B}=0$ as an initial condition, which implies $\Phi_{B}(\vec{x}) \approx 0$ for all time and hence corresponds to zero massive mode $\beta$. The solution (117) then reduces to the weak static limit of Einstein-Maxwell theory, as expected. The gravitational potential becomes $\Phi_{g}=\Phi_{m}$. The bumblebee field reduces to $\mathcal{E}_{\mu}=$ $\left(b \Phi_{m}, t \partial_{j}\left(\Phi_{q}+b \Phi_{m}\right)\right)$, which despite the appearance of the gravitational potential implies $\vec{E}=-\vec{\nabla} \Phi_{q}$ and the usual Gauss and Poisson equations $\vec{\nabla} \cdot \vec{E}=-\vec{\nabla}^{2} \Phi_{q}=$ $\rho_{q}$. We note in passing that conventional electrodynamics is also recovered in the limit $\left|M_{\beta}\right|^{2} \rightarrow \infty$ because taking this limit in Eq. (108) implies $\beta \rightarrow 0$ and hence $\Phi_{B} \rightarrow 0$. Thus, even with a nonzero massive mode $\beta$, a theory with a large mass parameter $\left|M_{\beta}\right|$ approximates EinsteinMaxwell theory in the low-energy regime, in agreement with the discussion following Eq. (99).

Other choices of initial conditions on $\Phi_{B}$ might appear in the context of a more fundamental theory for which a bumblebee model provides an effective partial low-energy theory. One set of examples with nonzero $\Phi_{B}$ consists of solutions with zero matter current, $J_{\mathrm{M}}^{\mu} \equiv 0$. Imposing $J_{\mathrm{M}}^{\mu} \equiv 0$ by hand is a common approach in the literature. It implies the bumblebee field has no couplings to matter and hence is unrelated to electrodynamics, being a field that interacts only gravitationally. The massless Lorentz NG modes then propagate freely as sterile fields that carry energy and momentum but convey no direct forces on charged particles. We emphasize here that a theory of this type can nonetheless lead to modifications of the gravitational interaction, contrary to some claims in the literature. This is readily illustrated in the context of the purely timelike example considered in this subsection. With $J_{\mathrm{M}}^{\mu} \equiv 0$, the linearized solutions for a static point particle of mass $m$ are still given by Eqs. (117), but $\Phi_{q}=$ 0 and the fields $\vec{E}$ and $\vec{B}$ are largely irrelevant. However, the gravitational potential $\Phi_{g}$ is modified by the potential $\Phi_{B}$ for the massive mode $\beta$, with the value of $\Phi_{B}$ fixed by initial conditions. 
In the absence of additional theoretical or experimental information, the possibilities for model building are vast. One could, for example, consider initial conditions with $\Phi_{B}$ proportional to $\Phi_{m}$,

$$
\Phi_{B}=\frac{\alpha}{4 \pi G b} \Phi_{m}
$$

with $\alpha$ a constant. For this class of solutions, $\Phi_{g}$ has the usual form $\Phi_{g}=-G^{*} m / r$ for a point mass but with a scaled value of the Newton gravitational constant

$$
G^{*}=(1-\alpha) G_{N}
$$

where $G_{N}$ is the value of $G^{*}$ for $\Phi_{B}=0$. This rescaling has no observable effects in the special observer frame because laboratory measurements would determine $G^{*}$ instead of $G_{N}$, although sidereal, annual, and solar motions might introduce detectable effects in realistic experiments. One could equally well consider instead examples in which $\Phi_{B}$ has nonlinear functional dependence on $\Phi_{m}$ or has other coordinate dependence. Various forms could be proposed as candidate models to explain phenomena such as dark matter or possibly dark energy. With the choice of $\Phi_{B}$ being conjecture at the level of the linearized equations, this approach is purely phenomenological. However, if a bumblebee model appears as part of the effective theory in a complete theory of quantum gravity, the form of $\Phi_{B}$ could well be fixed and predictions for dark matter and perhaps dark energy could become possible.

We conclude this subsection with some comments about the case where $b_{\mu}$ takes a more general timelike form in the given observer frame $O$. To investigate this, one can either study directly the weak static limit of the gravitational and bumblebee equations with a charged massive point particle at rest at the origin in $O$ as before, or one can boost the observer frame $O$ to another frame $O^{\prime}$ in which $b_{\mu}$ is purely timelike but the point particle is moving. The two pictures are related by observer Lorentz transformations and are therefore physically equivalent.

In the frame $O$ in which the particle is at rest, the matter energy-momentum tensor and current are given by Eq. (112) as before, but the bumblebee energy-momentum tensor $T_{B}^{\mu \nu}$ and current $J_{B}^{\mu}$ have additional nonzero components due to the nonzero spacelike components of $b_{\mu}$. The complete solution therefore involves components of $h_{\mu \nu}$ other than the gravitational potential $\Phi_{g}$. However, we can gain insight into the behavior of the massive mode by considering the constraint (89) and performing an observer Lorentz boost with velocity $\vec{v}_{B}=-\vec{b} / b_{0}$ to a frame $O^{\prime}$ in which $b_{\mu}$ becomes purely timelike. Since this transformation must reduce the constraint (89) to the purely timelike form (110), it follows that in the original frame $O$ the constraint takes the form

$$
\partial_{0} \beta+\vec{v}_{B} \cdot \vec{\nabla} \beta=0
$$

which implies

$$
\beta=\beta\left(\vec{x}-\vec{v}_{B} t\right)
$$

We see that the massive mode $\beta$ moves in the frame $O$ with a velocity $\vec{v}_{B}$ equal to the relative boost velocity linking $b_{\mu}$ to its purely timelike form. The gravitational and electrodynamic equations are therefore modified by timedependent source terms moving with velocity $\vec{v}_{B}$ relative to the point particle. An equivalent result is obtained by working directly in the frame $O^{\prime}$, in which $b_{\mu}$ is purely timelike and $\beta$ is therefore static, but in which all components of the matter energy-momentum tensor and current are nonzero and represent a particle moving with velocity $-\vec{v}_{B}$ relative to $\beta$. As before, the form of the massive mode $\beta$ is set by initial conditions. Since the equations are linear in either frame, the solutions consist of a superposition of conventional potentials and the massive-mode contributions with relative motion.

\section{Linear Lagrange-multiplier potential}

In this subsection, we discuss the KS bumblebee model (26) with the linear Lagrange-multiplier potential (6) and the definition (22):

$$
V=V_{L}(\lambda, X)=\lambda\left(B_{\mu} g^{\mu \nu} B_{\nu} \pm b^{2}\right) .
$$

Bumblebee models with this potential have been widely studied at the linearized level for about two decades [3]. Here, we compare and contrast this theory to the case with the smooth quadratic potential $V_{S}$. We show the Lagrange multiplier $\lambda$ produces effects in the $V_{L}$ model that are very similar to those of the massive mode $\beta$ in the $V_{S}$ model. Paralleling the treatment of the $V_{S}$ model in Sec. IV B, the mode expansion $B_{\mu}=b_{\mu}+\mathcal{E}_{\mu}$ of Eq. (60) in a Minkowski background is adopted, and the fields $h_{\mu \nu}$ and $\mathcal{E}_{\mu}$ are assumed weak so that linearization can be performed.

Variation of the action produces the gravitational and bumblebee equations of motion (72) and (77), together with the Lagrange-multiplier constraint

$$
X=B_{\mu} g^{\mu \nu} B_{\nu} \pm b^{2}=0 .
$$

This condition enforces the vanishing of the massive mode, as discussed in Sec. II B, which leaves only the Lorentz NG modes as possible excitations of the bumblebee field $B_{\mu}$. However, there is also an additional degree of freedom, the Lagrange multiplier $\lambda$ itself, that appears in the equations of motion.

One might naively expect the $V_{L}$ model to yield solutions identical to those obtained in the infinite-mass limit $\left|M_{\beta}\right| \rightarrow \infty$ of the $V_{S}$ model. For example, one might reason 
that an infinite mass would make energetically impossible any field excitations away from the minimum of $V_{S}$, leading to the constraint (126). However, the potential $V_{L}$ is a function of two combinations of fields, $V_{L}=V_{L}(\lambda, X)$, whereas $V_{S}(X)$ involves only one. The infinite-mass limit indeed suppresses $X$ excitations away from $X=0$ in $V_{S}$, but it contains no match for $\lambda$. There is thus an extra field degree of freedom in $V_{L}$ relative to the infinite-mass limit of $V_{S}$. For example, $V_{S}^{\prime} \rightarrow 0$ in the infinite-mass limit because no excitations of $X$ are allowed, whereas $V_{L}^{\prime}=$ $\lambda$, which need not vanish. We therefore conclude that the correspondence between the infinite-mass limit of the $V_{S}$ model and the $V_{L}$ model can occur only when $\lambda=0$.

To gain intuition about the effects associated with the Lagrange-multiplier field $\lambda$, consider its role in the equations of motion. Since the constraint (126) ensures no massive excitations can appear, the bumblebee energymomentum tensor (74) reduces to

$$
T_{B}^{\mu \nu}=T_{\mathrm{EM}}^{\mu \nu}+T_{V}^{\mu \nu}
$$

We find

$$
T_{V}^{\mu \nu}=2 \lambda B^{\mu} B^{\nu} \approx 2 b^{\mu} b^{\nu} \lambda,
$$

where the last form is the leading-order contribution in the linearized limit. Similarly, the bumblebee current (79) becomes

$$
J_{B}^{\mu}=-2 \lambda B^{\mu} \approx-2 b^{\mu} \lambda .
$$

Conservation of the matter current $J_{M}^{\mu}$ implies the constraint

$$
b^{\mu} \partial_{\mu} \lambda \approx 0 .
$$

When $\lambda \rightarrow 0$, all these equations reduce to those of Einstein-Maxwell theory, in agreement with the discussion of bumblebee electrodynamics in Sec. IVA 3. Moreover, by comparison with Eqs. (87)-(89), we see that the Lagrange multiplier $\lambda$ in the $V_{L}$ model plays a role very comparable to that of the massive mode $\beta$ in the $V_{S}$ model. In effect, $\lambda$ acts as an additional source of energymomentum density and current density in the equations of motion.

The propagating modes for the KS bumblebee model with the linear Lagrange-multiplier potential $V_{L}$ have been investigated elsewhere [3,5]. The usual two graviton modes propagate as free transverse massless modes independently of $\lambda$, as do the usual two photon modes emerging from the Lorentz NG modes. Since $\lambda$ has no kinetic terms, it is auxiliary and cannot propagate.

The weak static limit of the $V_{L}$ model can also be studied, following the lines of the discussion for the $V_{S}$ model in Sec. IV B 3. Consider, for example, the case of purely timelike $b_{\mu}=(b, 0,0,0)$. The constraint (130) then implies that $\lambda$ must be time independent at leading order,

$$
\partial_{0} \lambda \approx 0 \text {. }
$$

A nonzero $\lambda$ therefore acts as an additional static source of energy density and charge density, which can modify the static potentials. We again adopt the transverse gauge (107), for which the gravitational potential is $\Phi_{g}=$ $-h_{00} / 2$. Linearizing the constraint (126) for the purely timelike case yields

$$
\mathcal{E}_{0}-b \Phi_{g}=0
$$

As expected, this condition corresponds in the context of the $V_{S}$ model to enforcing the vanishing of the massive mode $\beta$. In the context of Einstein-Maxwell theory, it corresponds as before to a gauge-fixing condition that reduces to the usual temporal gauge in the absence of gravity.

For a single point particle of charge $q$ and mass $m$ at rest at the origin, the energy density $\rho_{m}$ and charge density $\rho_{q}$ are given by Eq. (112). The equations of motion in the weak static limit become

$$
\begin{aligned}
\nabla^{2} \Phi_{g} & \approx 4 \pi G\left(\rho_{m}+T_{\mathrm{EM}}^{00}+2 b^{2} \lambda\right), \\
\vec{\nabla}^{2} \mathcal{E}_{0}-\vec{\nabla} \cdot \partial_{0} \overrightarrow{\mathcal{E}} & \approx \rho_{q}+2 b \lambda, \\
\square \overrightarrow{\mathcal{E}}-\vec{\nabla}(\vec{\nabla} \cdot \overrightarrow{\mathcal{E}}) & \approx 0 .
\end{aligned}
$$

Comparison of these expressions with Eqs. (108) for the $V_{S}$ model again reveals the correspondence between the roles of the Lagrange-multiplier field $\lambda$ and the massive mode $\beta$.

With $\lambda$ acting as an effective source of charge and energy density, we can introduce a potential $\Phi_{B}$,

$$
\lambda \equiv-\frac{1}{2 b} \nabla^{2} \Phi_{B}
$$

which equivalently can be viewed as the solution to the Poisson equation

$$
\nabla^{2} \Phi_{B}=-\rho_{B}, \quad \rho_{B} \equiv 2 b \lambda .
$$

As before, let $\Phi_{m}$ denote the usual gravitational potential obeying Eq. (113), and let $\Phi_{q}$ denote the usual Coulomb potential obeying Eq. (114). In terms of these three potentials, the weak-field static solutions to the equations of motion (133) can be written as

$$
\begin{aligned}
\Phi_{g} & =\Phi_{m}-4 \pi G b \Phi_{B}, \\
\mathcal{E}_{0} & =b \Phi_{m}-4 \pi G b^{2} \Phi_{B}, \\
\mathcal{E}_{j} & =t \partial_{j}\left[\Phi_{q}+b \Phi_{m}+\left(1-4 \pi G b^{2}\right) \Phi_{B}\right] .
\end{aligned}
$$

The associated static gravitational field $\vec{g}$, static electric field $\vec{E}$, and static magnetic field $\vec{B}$ are all given by the 
same mathematical expressions as Eqs. (118), but the potential $\Phi_{B}$ is now defined in terms of $\lambda$ according to Eq. (134). Similarly, a nonzero Lagrange-multipler field $\lambda$ yields the same modified mathematical form (119) of the Gauss law, but the potential $\Phi_{B}$ and charge $\rho_{B}$ are now defined in terms of $\lambda$.

The bumblebee potential $\Phi_{B}$ in Eqs. (136) is undetermined by the equations of motion (133) and must be specified as an initial value. This specification also fixes the initial value of the Lagrange multiplier $\lambda$, and the condition (131) then ensures that $\lambda$ remains unchanged for all time. The situation here parallels that of the $V_{S}$ model, where initial conditions must be imposed that subsequently fix the bumblebee potential and the massive mode $\beta$ for all time. These results are special cases of a more general fact often overlooked in the literature: to be well defined, all bumblebee models require explicit initial conditions on the massive modes and Lagrange-multiplier fields. The subsequent development of the massive modes and Lagrange multipliers can then be deduced from the equations of motion or from derived constraints. In the absence of specified initial conditions, physical interpretations and predictions cannot be reliable. Moreover, in the absence of direct experimental observation or prediction from an underlying theory, the choice of initial conditions is largely unrestricted and can lead to widely differing effects.

One natural initial condition is $\Phi_{B}=0, \lambda=0$. The above results then reduce to Einstein-Maxwell theory in the weak static limit, as expected. This $V_{L}$ model has only field excitations maintaining both $V_{L}=0$ and $V_{L}^{\prime}=0$. It corresponds to the infinite-mass limit of $V_{S}$, which is itself equivalent to Einstein-Maxwell theory.

Another possibility is to consider models without direct coupling to matter, $J_{\mathrm{M}}^{\mu} \equiv 0$, which implies $\rho_{q} \equiv 0, \Phi_{q} \equiv$ 0 in the above equations. Like their $V_{S}$ counterparts discussed in Sec. IV B 3, these models are purely gravitational and are unrelated to electrodynamics. As always, initial conditions on $\Phi_{B}$ must be specified. One simple possibility, for example, is to choose $\Phi_{B}=-b \Phi_{g}$. This $V_{L}$ model is contained in the analysis of Ref. [47]. The spatial components of the bumblebee vanish, $\mathcal{E}_{j}=0$, so the bumblebee field $B_{\mu}$ is parallel to a timelike Killing vector. The modifications to $\Phi_{g}$ in this example involve a rescaling of the Newton gravitational constant.

Examples with both $\Phi_{B}$ and $\Phi_{q}$ nonzero can also be considered. These incorporate direct charge couplings to matter, but the static weak-field limit yields modified gravitational and Coulomb potentials given by Eq. (136). One simple example is the choice $\Phi_{B}=-\Phi_{q}$. Since $\Phi_{g} \neq \Phi_{m}$, this $V_{L}$ model has a modified gravitational potential. The solution for the bumblebee field has the form of a total derivative, $\mathcal{E}_{\mu}=\partial_{\mu}\left(t b \Phi_{g}\right)$. In the limit $q \rightarrow 0$ and $\lambda \rightarrow 0$, it provides an example of a solution that with $\lambda=0$ and hence $\Phi_{B}=0$ has been identified in
Ref. [45] as potentially flawed due to the formation of shock discontinuities in $\mathcal{E}_{\mu}$. Whether or not $\lambda$ is zero, this solution is unusual. Both the field strength $F_{\mu \nu}$ and the energy-momentum tensor $T_{\mathrm{EM}}^{\mu \nu}$ vanish because the effective charge density $\rho_{B}$ associated with the Lagrangemultiplier field cancels the matter charge density $\rho_{q}$. Although the field strengths are zero, the bumblebee field $\mathcal{E}_{\mu}$ must be nonzero because the constraint (132) implies $\mathcal{E}_{0}=b \Phi_{g}$, which cannot vanish in the presence of gravity. For a point charge the solution $\mathcal{E}_{\mu}$ does indeed contain a singularity, but this is physically unremarkable as it merely reflects the usual $1 / r$ dependence in $\Phi_{g}$. The same behavior arises in the standard solutions of Einstein-Maxwell theory in a gauge fixed by Eq. (132), to which the $V_{L}$ model is equivalent.

\section{Quadratic Lagrange-multiplier potential}

As a final example, we consider in this subsection the specific KS bumblebee model (26) with the quadratic Lagrange-multiplier potential (7)

$$
V=V_{Q}(\lambda, X)=\frac{1}{2} \lambda\left(B_{\mu} g^{\mu \nu} B_{\nu} \pm b^{2}\right)^{2},
$$

where $X$ is defined in Eq. (22). As before, we adopt the mode expansion $B_{\mu}=b_{\mu}+\mathcal{E}_{\mu}$ of Eq. (60) in a Minkowski background, and where useful we assume weak fields $h_{\mu \nu}$ and $\mathcal{E}_{\mu}$.

Bumblebee models with a quadratic Lagrangemultiplier potential have not previously been considered in detail in the literature. We introduce them here partly as a foil for the $V_{L}$ case, in which the Lagrange multiplier plays a key role in the physics of the model. In contrast, the Lagrange multiplier for the potential $V_{Q}$ decouples entirely from the classical dynamics. The point is that variation with respect to $\lambda$ yields the constraint $X^{2}=0$, which is equivalent to $X=0$ and forces the massive mode to vanish. However, the quadratic nature of $V_{Q}$ means that $\lambda$ always appears multiplied by $X$ in the gravitational and bumblebee field equations, so the on-shell condition $X=0$ forces the field $\lambda$ to decouple. Also, the potential obeys both $V_{Q}=0$ and $V_{Q}^{\prime}=0$ on shell, so the bumblebee energy-momentum tensor and bumblebee current reduce to the standard expressions for electrodynamics in curved spacetime. The equations of motion are therefore equivalent to those of Einstein-Maxwell electrodynamics in the gauge $X=0$. This correspondence holds both in the linearized limit and in the full nonlinear theory.

Since the equations of motion generated by a theory with a quadratic Lagrange-multiplier potential are the equations in the absence of the constraint plus the constraint itself, introducing this type of potential is equivalent at the classical level to imposing the constraint by hand on the equations of motion. The only distinction between the two approaches is the presence of the decoupled Lagrange-multiplier field. Bumblebee models with the 
potential $V_{Q}$ therefore incorporate a variety of models in which constraints are imposed by hand. For example, Will and Nordtvedt have considered solutions involving a nonzero background value for a vector in bumblebee-type models with Lagrange density of the form (19) but without a potential term [42]. This approach produces equations of motion identical to those of the corresponding $V_{Q}$ model.

The $V_{Q}$ models are also related to theories in which the constraint is substituted into the action before varying, although the correspondence is inexact. For example, Nambu has investigated the Maxwell action in Minkowski spacetime using the constraint $X=0$ for the purely timelike case as a nonlinear gauge condition substituted into the action prior to the variation [53]. This represents gauge fixing at the level of the action, and it yields a total of four equations for the fields: the original constraint and three equations of motion from the variation. However, the corresponding $V_{Q}$ model yields five equations of motion instead, one of which is the constraint. The extra equation is the Gauss law, which in the Nambu approach is imposed as a separate initial condition that subsequently holds at all times by virtue of the three equations of motion and the constraint.

For the specific KS bumblebee model (26) with potential $V_{Q}$ in Eq. (137), the freely propagating modes can be found by linearizing the gravitational and bumblebee equations of motion (72) and (77) and the constraint $X=0$. The linearization generates the same equations as emerge for the $V_{L}$ model with potential (125) in the limit $\lambda \rightarrow 0$. The propagating modes consist of the usual graviton and photon in an axial gauge [5], as expected. Similarly, the weak static limit of the $V_{Q}$ model with potential (137) produces equations identical at linear level to those of the $V_{L}$ model with potential (125) in the limit $\lambda \rightarrow 0$. The usual gravitational and electromagnetic Poisson equations therefore emerge, and the correct Newton and Coulomb potentials hold at the linearized level.

The exact correspondence between the static limit of the $V_{Q}$ bumblebee model and the Newton and Coulomb potentials involves the nonlinear constraint (126). The explicit forms of the solutions are therefore also nonlinear. For example, consider again the case of a point particle of mass $m$ and charge $q$ at rest at the origin in the presence of a purely timelike vacuum value $b_{\mu}=(b, 0,0,0)$. The equation of motion for $\lambda$ is the constraint (126), which represents a nonlinear condition relating $\mathcal{E}_{\mu}$ and $h_{\mu \nu}$. Expanding this constraint through quadratic order gives

$$
\mathcal{E}_{0}^{2}+2 b \mathcal{E}_{0}-2 b^{2} \Phi_{g}-4 b \Phi_{m} \mathcal{E}_{0}-\mathcal{E}_{j}^{2} \approx 0,
$$

where $\Phi_{m}$ obeys the conventional Poisson equation (113) for the point source with mass and charge density given by Eq. (112). In the transverse gauge, the gravitational potential $\Phi_{g}$ obeys

$$
\vec{\nabla}^{2} \Phi_{g} \approx 4 \pi G\left(\rho_{m}+T_{\mathrm{EM}}^{00}\right),
$$

where the energy-momentum tensor $T_{\mathrm{EM}}^{00}$ has the usual Einstein-Maxwell form (84). The solution for $\Phi_{g}$ is therefore the standard gravitational potential for a static point charge in a curved spacetime in the weak-field limit. At quadratic order, the solution $\mathcal{E}_{\mu}=\left(\mathcal{E}_{0}, \mathcal{E}_{j}\right)$ satisfying the constraint (138) and the field equations is found to be

$$
\begin{aligned}
\mathcal{E}_{0} \approx & b \Phi_{g}+\frac{3 b}{2} \Phi_{g}^{2}+\frac{t^{2}}{2 b}\left[\partial_{j}\left(\Phi_{q}+b \Phi_{g}\right)\right]^{2}, \\
\mathcal{E}_{j} \approx & t \partial_{j}\left(\Phi_{q}+b \Phi_{g}\right)+3 b t \Phi_{g} \partial_{j} \Phi_{g} \\
& +\frac{t^{3}}{3 b}\left[\partial_{k}\left(\Phi_{q}+b \Phi_{g}\right)\right] \partial_{j} \partial_{k}\left(\Phi_{q}+b \Phi_{g}\right),
\end{aligned}
$$

where $\Phi_{q}$ is the conventional Coulomb potential obeying the Poisson equation (114). These expressions are both time dependent and nonlinear, but they nonetheless generate the usual static electric field $\vec{E}$ and magnetic field $\vec{B}$ for a point charge. Explicit calculation to quadratic order yields

$$
\vec{E} \approx-\vec{\nabla} \Phi_{q}, \quad \vec{B} \approx 0,
$$

which implies the usual form $\vec{\nabla} \cdot \vec{E} \approx \rho_{q}$ of the Gauss law. From these equations, we see again that Einstein-Maxwell theory is recovered despite the absence of U(1) symmetry. In effect, the nonlinear condition (138) plays the role of a nonlinear gauge-fixing condition in a U(1)-invariant theory, removing a degree of freedom and leaving only $\mathrm{NG}$ modes that propagate as photons and generate the usual Coulomb potential in the weak-field limit.

\section{SUMMARY}

In this paper, we have investigated the properties of the massive modes that can emerge from spontaneous local Lorentz and diffeomorphism breaking. In Riemann spacetime, no massive modes of the conventional Higgs-type can appear because covariant kinetic terms involve connections with derivatives. However, an alternative form of the Higgs mechanism can occur instead, in which massive modes originate from quadratic couplings in the potential $V$ inducing the symmetry breaking.

Section II provides an analysis of this alternative Higgs mechanism in the general context of an arbitrary tensor field. Both smooth potentials and Lagrange-multiplier potentials are considered. Massive modes appear for a smooth potential when excitations with $V^{\prime} \neq 0$ exist, and they are formed as combinations of field and metric fluctuations. For Lagrange-multiplier potentials the massive modes are constrained to vanish, but the Lagrange-multiplier fields can play a related role. The propagation of the massive modes depends on the nature of the kinetic terms in the theory, and the requirements of unitarity and ghost-free propagation constrain possible models. Even if the massive modes are nonpropagating they can influence gravitational phenomena through, for example, effects on the static 
gravitational potential. These modifications are of potential interest in alternative theories of gravity and descriptions of phenomena such as dark matter or dark energy.

Following the general treatment, we investigate in Sec. III a broad class of theories called bumblebee models that involve gravitationally coupled vector fields with spontaneous local Lorentz and diffeomorphism breaking. For arbitrary quadratic kinetic terms, the Lagrange density is given in Eq. (19). Along with the symmetry-breaking potential $V$ and a matter sector, this Lagrange density involves five parameters, four of which can be linearly independent in specific models. A particularly attractive class of theories are the KS bumblebee models, which have kinetic term of the Maxwell form and hence an additional constraint that minimizes problems with unitarity and ghosts. These models also offer candidate alternatives to Einstein-Maxwell electrodynamics.

In a series of subsections and the associated appendix, we provide some results valid for general bumblebee models. The observer and particle forms of local Lorentz transformations and diffeomorphisms are presented. Using the vierbein formalism, some decompositions of the bumblebee field and metric are given that are suitable for the identification of Lorentz and diffeomorphism NG modes. The effects of various choices of Lorentz and diffeomorphism gauges are described. Alternative decompositions used in some of the literature are also discussed, in which the Lorentz NG modes are hidden and only spacetime variables are used.

To provide explicit examples and to gain insight via a more detailed analysis, we focus attention in Sec. IV on the class of KS bumblebee models. The basic equations of motion and conservation laws are obtained, and some properties of the bumblebee currents are considered. The interpretation of these bumblebee models as theories of electromagnetism and gravity, known as bumblebee electrodynamics, is discussed. They contain four transverse massless modes, two of which are massless gravitons and two of which are massless photons, along with a massive mode or Lagrange-multiplier mode. When the massive mode or Lagrange multipler vanishes, or in the limit of infinite mass, conventional Einstein-Maxwell theory in an axial gauge is recovered.

Section IV also contains subsections considering in more detail various types of potentials, including the smooth potential $V_{S}$ in Eq. (86), the linear Lagrange-multiplier potential $V_{L}$ in Eq. (125), and the quadratic Lagrangemultiplier potential $V_{Q}$ in Eq. (137). For the $V_{S}$ model, the gravitational and bumblebee equations of motion are investigated to determine whether a physical massive mode can propagate as a free field. The sign of the squared-mass term depends on whether the bumblebee vacuum value $b_{\mu}$ is timelike or spacelike. In the timelike case, the massive mode is a ghost, while in the spacelike case the squared mass has the usual sign. However, in both cases the dis- persion law is unconventional and no localized physical solutions satisfying suitable asymptotic boundary conditions exist, so the $V_{S}$ model has no freely propagating massive modes. Nonetheless, the massive mode has a physical impact as an auxiliary field, acting as an additional source of energy-momentum density and current density. In the weak static limit, for example, the solutions to the equations of motion in the presence of mass and charge describe modified Newton and Coulomb potentials according to Eq. (117). These may be of phenomenological interest in the context of dark matter and perhaps also dark energy. The effects are controlled by the massive mode, but its form is dynamically undetermined and must be imposed via initial conditions.

Many of the results obtained for the $V_{S}$ model apply also to the $V_{L}$ and $V_{Q}$ models that have Lagrange-multiplier fields. For example, all the models contain four massless propagating modes behaving like gravitons and photons. Although the $V_{L}$ and $V_{Q}$ models generate an additional constraint that eliminates the massive mode on shell, the Lagrange-multiplier field appears instead as a extra degree of freedom playing a similar role to that of the massive mode in the $V_{S}$ model. The form of the Lagrange multiplier must be set by initial conditions, and if it is chosen to vanish then Einstein-Maxwell theory is recovered. The key difference between the $V_{L}$ and $V_{Q}$ models lies in the role of the Lagrange multiplier, which can affect the physics as an auxiliary mode in the $V_{L}$ model but which decouples from the theory in the $V_{Q}$ model.

In the context of bumblebee electrodynamics, the massive mode or Lagrange-multiplier field acts as an additional degree of freedom relative to Einstein-Maxwell theory. The extra freedom arises because the bumblebee model has no U(1) gauge symmetry, and the structure of the kinetic terms implies that the freedom must be fixed as an initial condition. In the absence of experimental evidence for a massive mode or of guidance from an underlying theory, the choice of initial condition is largely arbitrary. A natural choice sets the massive mode or Lagrange multiplier field to zero, reducing the theory to Einstein-Maxwell electrodynamics up to possible SME matter-sector couplings. Bumblebee electrodynamics therefore provides a candidate alternative explanation for the existence of massless photons, based on the masslessness of NG modes instead of the usual gauge symmetry. In any case, the possibility of spontaneous breaking of local Lorentz and diffeomorphism symmetry remains a promising avenue for exploring physics emerging from the Planck scale.

\section{ACKNOWLEDGMENTS}

This work was supported in part by DOE Grant No. DEFG02-91ER40661, NASA Grant No. NAG3-2914, and NSF Grant No. PHY-0554663. 


\section{APPENDIX: TRANSFORMATIONS}

In this appendix, we provide explicit transformation formulas for the vierbein, bumblebee, and NG field components under particle diffeomorphisms, particle local Lorentz transformations, observer general coordinate transformations, and observer local Lorentz transformations. The vierbein decomposition is given in Eq. (37), the bumblebee decomposition is given in Eq. (40), and the NG fields are defined in Eq. (47). The transformation formulas can be deduced from these expressions and from the behavior of the vacuum expectation values. All the formulas given here are linearized assuming infinitesimal fields and transformation parameters.

\section{Particle transformations}

Under infinitesimal particle diffeomorphisms,

$$
\begin{aligned}
b_{\mu} & \rightarrow b_{\mu}, \\
\eta_{\mu \nu} & \rightarrow \eta_{\mu \nu}, \\
\delta^{\mu}{ }_{\nu} & \rightarrow \delta^{\mu}{ }_{\nu}, \\
\beta_{\mu}^{\mathrm{t}} & \rightarrow \beta_{\mu}^{\mathrm{t}}, \\
\beta & \rightarrow \beta, \\
h_{\mu \nu} & \rightarrow h_{\mu \nu}-\partial_{\mu} \xi_{\nu}-\partial_{\nu} \xi_{\mu}, \\
\chi_{\mu \nu} & \rightarrow \chi_{\mu \nu}-\frac{1}{2}\left(\partial_{\mu} \xi_{\nu}-\partial_{\nu} \xi_{\mu}\right) \\
e_{\mu \nu} \equiv \eta_{\nu \alpha} e_{\mu}{ }^{\alpha} & \rightarrow e_{\mu \nu}-\partial_{\mu} \xi_{\nu} \\
e^{\mu \nu} \equiv \eta^{\nu \alpha} e^{\mu}{ }_{\alpha} & \rightarrow e^{\mu \nu}+\partial^{\nu} \xi^{\mu}, \\
B_{\mu} & \rightarrow B_{\mu}-\left(\partial_{\mu} \xi_{\nu}\right) b^{\nu}, \\
B^{\mu} & \rightarrow B^{\mu}+\left(\partial_{\nu} \xi^{\mu}\right) b^{\nu}, \\
\Xi_{\mu} & \rightarrow \Xi_{\mu}+\xi_{\mu}, \\
\mathcal{E}_{\mu \nu} & \rightarrow \mathcal{E}_{\mu \nu} .
\end{aligned}
$$

Under infinitesimal local particle Lorentz transformations,

$$
\begin{aligned}
b_{\mu} & \rightarrow b_{\mu}, \\
\eta_{\mu \nu} & \rightarrow \eta_{\mu \nu}, \\
\delta^{\mu}{ }_{\nu} & \rightarrow \delta^{\mu}{ }_{\nu}, \\
\beta_{\mu}^{\mathrm{t}} & \rightarrow \beta_{\mu}^{\mathrm{t}}+\epsilon_{\mu \nu} b^{\nu}, \\
\beta & \rightarrow \beta, \\
h_{\mu \nu} & \rightarrow h_{\mu \nu}, \\
\chi_{\mu \nu} & \rightarrow \chi_{\mu \nu}-\epsilon_{\mu \nu} \\
e_{\mu \nu} \equiv \eta_{\nu \alpha} e_{\mu}{ }^{\alpha} & \rightarrow e_{\mu \nu}-\epsilon_{\mu \nu} \\
e^{\mu \nu} \equiv \eta^{\nu \alpha} e^{\mu}{ }_{\alpha} & \rightarrow e^{\mu \nu}-\epsilon^{\mu \nu}, \\
B_{\mu} & \rightarrow B_{\mu}, \\
\Xi_{\mu} & \rightarrow \Xi_{\mu}, \\
\mathcal{E}_{\mu \nu} & \rightarrow \mathcal{E}_{\mu \nu}+\epsilon_{\mu \nu} .
\end{aligned}
$$

\section{Observer transformations}

The primary difference between observer and particle transformations is that the components of vacuum-valued fields transform under observer transformations. However, additional differences arise when the distinction between spacetime and local indices is dropped. In particular, care is required in expressing observer transformation laws for the metric and the vierbein.

Three versions of the Minkowski metric appear in the formalism: the spacetime metric $\eta_{\mu \nu}$, the local metric $\eta_{a b}$, and the mixed metric $\eta_{\mu a}$ used with the vierbein. All three are numerically equal in cartesian coordinates, but they behave differently under observer transformations. In the customary notation using only Greek indices, these three metrics must be labeled differently. Since they represent vacuum values of the metric, we write them as expectation values and define

$$
\begin{aligned}
& \left\langle\eta_{\mu \nu}\right\rangle_{\eta}=\left.\left(\eta_{a b}\right)\right|_{a b \rightarrow \mu \nu}, \\
& \left\langle\eta_{\mu \nu}\right\rangle_{e}=\left.\left(\eta_{\mu a}\right)\right|_{a \rightarrow \nu}, \\
& \left\langle\eta_{\mu \nu}\right\rangle_{g} \equiv \eta_{\mu \nu} .
\end{aligned}
$$

The inverse metrics are denoted with upper indices and are defined so that

$$
\left\langle\eta_{\mu \alpha}\right\rangle_{g}\left\langle\eta^{\alpha \nu}\right\rangle_{g} \approx\left\langle\eta_{\mu \alpha}\right\rangle_{e}\left\langle\eta^{\alpha \nu}\right\rangle_{e} \approx\left\langle\eta_{\mu \alpha}\right\rangle_{\eta}\left\langle\eta^{\alpha \nu}\right\rangle_{\eta} \approx \delta_{\mu}^{\nu} .
$$

In terms of these vacuum values, the metric and its inverse involve infinitesimal excitations:

$$
\begin{aligned}
& g_{\mu \nu} \approx\left\langle\eta_{\mu \nu}\right\rangle_{g}+h_{\mu \nu}, \\
& g^{\mu \nu} \approx\left\langle\eta^{\mu \nu}\right\rangle_{g}-h^{\mu \nu} .
\end{aligned}
$$

The transformation laws for the vierbein also depend on the component basis. We define

$$
\begin{aligned}
& \left\langle e_{\mu \nu}\right\rangle \approx\left\langle\eta_{\mu \nu}\right\rangle_{e}+\left\langle\chi_{\mu \nu}\right\rangle, \\
& \left\langle e^{\mu \nu}\right\rangle \approx\left\langle\eta^{\mu \nu}\right\rangle_{e}+\left\langle\chi^{\mu \nu}\right\rangle, \\
& \left\langle e^{\mu}{ }_{\nu}\right\rangle \approx\left\langle\eta_{\nu \alpha}\right\rangle_{\eta}\left\langle e^{\mu \alpha}\right\rangle, \\
& \left\langle e_{\mu}{ }^{\nu}\right\rangle \approx\left\langle\eta^{\nu \alpha}\right\rangle_{\eta}\left\langle e_{\mu \alpha}\right\rangle .
\end{aligned}
$$

For example, these vacuum values obey

$$
\left\langle\eta_{\mu \nu}\right\rangle_{g}=\left\langle e_{\mu \alpha}\right\rangle\left\langle e_{\nu \beta}\right\rangle\left\langle\eta^{\alpha \beta}\right\rangle_{\eta}=\left\langle\eta_{\mu \alpha}\right\rangle_{e}\left\langle\eta_{\nu \beta}\right\rangle_{e}\left\langle\eta^{\alpha \beta}\right\rangle_{\eta},
$$

as expected from the relationship between the metric and the vierbein. The vierbein field itself can be written

$$
\begin{aligned}
& e_{\mu \nu} \approx\left\langle\eta_{\mu \nu}\right\rangle_{e}+\left\langle\chi_{\mu \nu}\right\rangle+\frac{1}{2} h_{\mu \nu}+\chi_{\mu \nu}, \\
& e^{\mu \nu} \approx\left\langle\eta^{\mu \nu}\right\rangle_{e}+\left\langle\chi^{\mu \nu}\right\rangle-\frac{1}{2} h^{\mu \nu}+\chi^{\mu \nu} .
\end{aligned}
$$

In much of the literature, the vacuum value for $\left\langle\chi_{\mu \nu}\right\rangle$ is assumed to vanish in cartesian coordinates. However, this choice is observer dependent. Under either observer gen- 
eral coordinate transformations or observer local Lorentz transformations, a zero value of $\left\langle\chi_{\mu \nu}\right\rangle$ transforms into a nonzero value. We therefore include the vacuum value $\left\langle\chi_{\mu \nu}\right\rangle$ in the formulas below.

The transformation rules for infinitesimal observer general coordinate transformations are

$$
\begin{aligned}
\left\langle\eta_{\mu \nu}\right\rangle_{g} & \rightarrow\left\langle\eta_{\mu \nu}\right\rangle_{g}-\partial_{\mu} \xi_{\nu}-\partial_{\nu} \xi_{\mu}, \\
\left\langle\eta^{\mu \nu}\right\rangle_{g} & \rightarrow\left\langle\eta^{\mu \nu}\right\rangle_{g}+\partial^{\mu} \xi^{\nu}+\partial^{\nu} \xi^{\mu}, \\
\left\langle\eta_{\mu \nu}\right\rangle_{e} & \rightarrow\left\langle\eta_{\mu \nu}\right\rangle_{e}-\frac{1}{2}\left(\partial_{\mu} \xi_{\nu}+\partial_{\nu} \xi_{\mu}\right), \\
\left\langle\eta^{\mu \nu}\right\rangle_{e} & \rightarrow\left\langle\eta^{\mu \nu}\right\rangle_{e}+\frac{1}{2}\left(\partial^{\mu} \xi^{\nu}+\partial^{\nu} \xi^{\mu}\right), \\
\left\langle\eta_{\mu \nu}\right\rangle_{\eta} & \rightarrow\left\langle\eta_{\mu \nu}\right\rangle_{\eta}, \\
\left\langle\eta^{\mu \nu}\right\rangle_{\eta} & \rightarrow\left\langle\eta^{\mu \nu}\right\rangle_{\eta}, \\
\left\langle\chi_{\mu \nu}\right\rangle & \rightarrow\left\langle\chi_{\mu \nu}\right\rangle-\frac{1}{2}\left(\partial_{\mu} \xi_{\nu}-\partial_{\nu} \xi_{\mu}\right), \\
\left\langle\chi^{\mu \nu}\right\rangle & \rightarrow\left\langle\chi^{\mu \nu}\right\rangle-\frac{1}{2}\left(\partial^{\mu} \xi^{\nu}-\partial^{\nu} \xi^{\mu}\right), \\
\left\langle e_{\mu \nu}\right\rangle & \rightarrow\left\langle e_{\mu \nu}\right\rangle-\partial_{\mu} \xi_{\nu}, \\
\left\langle e^{\mu \nu}\right\rangle & \rightarrow\left\langle e^{\mu \nu}\right\rangle+\partial^{\nu} \xi^{\mu}, \\
b_{\mu} & \rightarrow b_{\mu}-\left(\partial_{\mu} \xi_{\nu}\right) b^{\nu}, \\
b^{\mu}=\left\langle\eta^{\mu \nu}\right\rangle_{g} b_{\nu} & \rightarrow b^{\mu}+\left(\partial_{\nu} \xi^{\mu}\right) b^{\nu}, \\
\beta_{\mu}^{\mathrm{t}} & \rightarrow \beta_{\mu}^{\mathrm{t}}, \\
\beta & \rightarrow \beta, \\
h_{\mu \nu} & \rightarrow h_{\mu \nu}, \\
\chi_{\mu \nu} & \rightarrow \chi_{\mu \nu}, \\
e_{\mu \nu} & \rightarrow e_{\mu \nu}-\partial_{\mu} \xi_{\nu}, \\
e^{\mu \nu} & \rightarrow e^{\mu \nu}+\partial^{\nu} \xi^{\mu}, \\
B_{\mu} & \rightarrow B_{\mu}-\left(\partial_{\mu} \xi_{\nu}\right) b^{\nu}, \\
B^{\mu} & \rightarrow B^{\mu}+\left(\partial_{\nu} \xi^{\mu}\right) b^{\nu}, \\
\Xi_{\mu} & \rightarrow \Xi_{\mu}, \\
\mathcal{E}_{\mu \nu} & \rightarrow \mathcal{E}_{\mu \nu} . \\
& \\
b^{\mu} & \\
b^{\mu} &
\end{aligned}
$$

The formulas for infinitesimal observer local Lorentz transformations are

$$
\begin{aligned}
\left\langle\eta_{\mu \nu}\right\rangle_{g} & \rightarrow\left\langle\eta_{\mu \nu}\right\rangle_{g}, \\
\left\langle\eta_{\mu \nu}\right\rangle_{e} & \rightarrow\left\langle\eta_{\mu \nu}\right\rangle_{e}, \\
\left\langle\eta_{\mu \nu}\right\rangle_{\eta} & \rightarrow\left\langle\eta_{\mu \nu}\right\rangle_{\eta}, \\
\left\langle\chi_{\mu \nu}\right\rangle & \rightarrow\left\langle\chi_{\mu \nu}\right\rangle-\epsilon_{\mu \nu}, \\
\left\langle e_{\mu \nu}\right\rangle & \rightarrow\left\langle e_{\mu \nu}\right\rangle-\epsilon_{\mu \nu}, \\
b_{\mu} & \rightarrow b_{\mu}+\epsilon_{\mu \nu} b^{\nu}, \\
\beta_{\mu}^{\mathrm{t}} & \rightarrow \beta_{\mu}^{\mathrm{t}}, \\
\beta & \rightarrow \beta, \\
h_{\mu \nu} & \rightarrow h_{\mu \nu}, \\
\chi_{\mu \nu} & \rightarrow \chi_{\mu \nu}, \\
e_{\mu \nu} & \rightarrow e_{\mu \nu}-\epsilon_{\mu \nu}, \\
B_{\mu} & \rightarrow B_{\mu}, \\
\Xi_{\mu} & \rightarrow \Xi_{\mu}, \\
\mathcal{E}_{\mu \nu} & \rightarrow \mathcal{E}_{\mu \nu} .
\end{aligned}
$$

In these expressions, indices can be raised and lowered using $\left\langle\eta_{\mu \nu}\right\rangle_{\eta}$.

For some purposes, it may be desirable for an observer transformation to take the appearance of the inverse of a particle transformation. This can be achieved by changing the signs of the parameters, $\xi_{\mu} \rightarrow-\xi_{\mu}$ and $\epsilon_{\mu \nu} \rightarrow-\epsilon_{\mu \nu}$, either in the formulas for the observer transformations or in those for the particle transformations. For example, with signs chosen appropriately the bumblebee field $B_{\mu}$ can be arranged to be invariant, $B_{\mu} \rightarrow B_{\mu}$, under the composite transformation consisting of a particle diffeomorphism followed by the corresponding inverse general coordinate transformation. However, the reader is cautioned that invariance of this type may fail for the vacuum value and excitations of $B_{\mu}$. For instance, the vacuum value $b_{\mu}$ is invariant under particle diffeomorphisms but transforms as ordinary components of a vector under observer general coordinate transformations.
[1] Y. Nambu, Phys. Rev. Lett. 4, 380 (1960); J. Goldstone, Nuovo Cimento 19, 154 (1961); J. Goldstone, A. Salam, and S. Weinberg, Phys. Rev. 127, 965 (1962).

[2] P. W. Anderson, Phys. Rev. 130, 439 (1963); P. W. Higgs, Phys. Lett. 12, 132 (1964); F. Englert and R. Brout, Phys. Rev. Lett. 13, 321 (1964); G. S. Guralnik, C. R. Hagen, and T. W. B. Kibble, Phys. Rev. Lett. 13, 585 (1964).

[3] V. A. Kostelecký and S. Samuel, Phys. Rev. D 40, 1886 (1989); Phys. Rev. Lett. 63, 224 (1989).
[4] V. A. Kostelecký, Phys. Rev. D 69, 105009 (2004).

[5] R. Bluhm and V. A. Kostelecký, Phys. Rev. D 71, 065008 (2005).

[6] V. A. Kostelecký and S. Samuel, Phys. Rev. D 39, 683 (1989); V. A. Kostelecký and R. Potting, Nucl. Phys. B359, 545 (1991).

[7] See, for example, I. Mocioiu, M. Pospelov, and R. Roiban, Phys. Lett. B 489, 390 (2000); S. M. Carroll et al., Phys. Rev. Lett. 87, 141601 (2001); Z. Guralnik, R. Jackiw, S. Y. 
Pi, and A.P. Polychronakos, Phys. Lett. B 517, 450 (2001); C.E. Carlson, C. D. Carone, and R. F. Lebed, Phys. Lett. B 518, 201 (2001); A. Anisimov, T. Banks, M. Dine, and M. Graesser, Phys. Rev. D 65, 085032 (2002); A. Das, J. Gamboa, J. Lopez-Sarrion, and F. A. Schaposnik, Phys. Rev. D 72, 107702 (2005).

[8] O. Bertolami, R. Lehnert, R. Potting, and A. Ribeiro, Phys. Rev. D 69, 083513 (2004); V. A. Kostelecký, R. Lehnert, and M. Perry, Phys. Rev. D 68, 123511 (2003); R. Jackiw and S.-Y. Pi, Phys. Rev. D 68, 104012 (2003).

[9] See, for example, G. Amelino-Camelia, C. Lämmerzahl, A. Macias, and H. Müller, in Gravitation and Cosmology: 2nd Mexican Meeting on Mathematical and Experimental Physics, edited by A. Macias et al., AIP Conf. Proc. No. 758 (AIP, New York, 2005), p. 30; N.E. Mavromatos, Lect. Notes Phys. 669, 245 (2005); H. A. Morales-Tecotl and L. F. Urrutia, in Particles and Fields: $X$ Mexican Workshop on Particles and Fields; and Commemorative Vol. of the Division of Particles and Fields of the Mexican Physical Society, edited by A. Bashir et al., AIP Conf. Proc. No. 857 (AIP, New York, 2006), p. 205; Y. Bonder and D. Sudarsky, arXiv:0709.0551.

[10] C. D. Froggatt and H. B. Nielsen, arXiv:hep-ph/0211106.

[11] J. D. Bjorken, Phys. Rev. D 67, 043508 (2003).

[12] See, for example, C. P. Burgess, J. Cline, E. Filotas, J. Matias, and G. D. Moore, J. High Energy Phys. 03 (2002) 043; A. R. Frey, J. High Energy Phys. 04 (2003) 012; J. Cline and L. Valcárcel, J. High Energy Phys. 03 (2004) 032.

[13] M. Berger and V. A. Kostelecký, Phys. Rev. D 65 , 091701(R) (2002); P. A. Bolokhov, S. G. Nibbelink, and M. Pospelov, Phys. Rev. D 72, 015013 (2005).

[14] V. A. Kostelecký and S. Samuel, Phys. Rev. D 42, 1289 (1990); Phys. Rev. Lett. 66, 1811 (1991).

[15] N. Arkani-Hamed, H.-C. Cheng, M. Luty, and S. Mukohyama, J. High Energy Phys. 05 (2004) 074; N. Arkani-Hamed, H.-C. Cheng, M. Luty, and J. Thaler, J. High Energy Phys. 07 (2005) 029; D. S. Gorbunov and S. M. Sibiryakov, J. High Energy Phys. 09 (2005) 082; H.-C. Cheng, M. Luty, S. Mukohyama, and J. Thaler, J. High Energy Phys. 05 (2006) 076; G. Dvali, O. Pujolas, and M. Redi, Phys. Rev. D 76, 044028 (2007).

[16] M. V. Libanov and V. A. Rubakov, J. High Energy Phys. 08 (2005) 001; S. Dubovsky, P. Tinyakov, and M. Zaldarriaga, J. High Energy Phys. 11 (2007) 083.

[17] V. A. Kostelecký and R. Potting, Phys. Rev. D 51, 3923 (1995).

[18] D. Colladay and V. A. Kostelecký, Phys. Rev. D 55, 6760 (1997); 58, 116002 (1998).

[19] For recent reviews of various experimental and theoretical approaches to Lorentz violation see, for example, CPT and Lorentz Symmetry I-IV, edited by V. A. Kostelecký (World Scientific, Singapore, 1999-2008); R. Bluhm, Lect. Notes Phys. 702, 191 (2006); D. M. Mattingly, Living Rev. Relativity 8, 5 (2005).

[20] A tabulation of results is given in V. A. Kostelecký and N. Russell, arXiv:0801.0287.

[21] J. Lipa et al., Phys. Rev. Lett. 90, 060403 (2003); H. Müller et al., Phys. Rev. Lett. 91, 020401 (2003); P. Wolf et al., Gen. Relativ. Gravit. 36, 2351 (2004); Phys. Rev. D 70, 051902 (2004); M. Tobar et al., Phys. Rev. D 71, 025004 (2005); S. Herrmann et al., Phys. Rev. Lett. 95, 150401 (2005); P. L. Stanwix et al., Phys. Rev. D 74, 081101(R) (2006); M. Hohensee et al., Phys. Rev. D 71, 025004 (2007); H. Müller et al., Phys. Rev. Lett. 99, 050401 (2007); S. Reinhardt et al., Nature Phys. 3, 861 (2007); S. M. Carroll, G. B. Field, and R. Jackiw, Phys. Rev. D 41, 1231 (1990); R. Jackiw and V. A. Kostelecký, Phys. Rev. Lett. 82, 3572 (1999); V. A. Kostelecký and M. Mewes, Phys. Rev. Lett. 87, 251304 (2001); Phys. Rev. D 66, 056005 (2002); Phys. Rev. Lett. 97, 140401 (2006); 99, 011601 (2007); Q. G. Bailey and V. A. Kostelecký, Phys. Rev. D 70, 076006 (2004); C. D. Carone, M. Sher, and M. Vanderhaeghen Phys. Rev. D 74, 077901 (2006); B. Altschul, Phys. Rev. Lett. 96, 201101 (2006); Phys. Rev. Lett. 98, 041603 (2007); Phys. Rev. D 75, 105003 (2007).

[22] H. Dehmelt et al., Phys. Rev. Lett. 83, 4694 (1999); R. Mittleman et al., Phys. Rev. Lett. 83, 2116 (1999); G. Gabrielse et al., Phys. Rev. Lett. 82, 3198 (1999); R. Bluhm et al., Phys. Rev. Lett. 82, 2254 (1999); Phys. Rev. Lett. 79, 1432 (1997); Phys. Rev. D 57, 3932 (1998); D. Colladay and V. A. Kostelecký, Phys. Lett. B 511, 209 (2001); B. Altschul, Phys. Rev. D 74, 083003 (2006); G. M. Shore, Nucl. Phys. B717, 86 (2005).

[23] B. Heckel et al., Phys. Rev. Lett. 97, 021603 (2006); L.-S. Hou, W.-T. Ni, and Y.-C.M. Li, Phys. Rev. Lett. 90, 201101 (2003); R. Bluhm and V. A. Kostelecký, Phys. Rev. Lett. 84, 1381 (2000).

[24] H. Müller, S. Herrmann, A. Saenz, A. Peters, and C. Lämmerzahl, Phys. Rev. D 70, 076004 (2004); H. Müller, Phys. Rev. D 71, 045004 (2005).

[25] D. Bear et al., Phys. Rev. Lett. 85, 5038 (2000); D. F. Phillips et al., Phys. Rev. D 63, 111101 (2001); M. A. Humphrey et al., Phys. Rev. A 68, 063807 (2003); F. Canè et al., Phys. Rev. Lett. 93, 230801 (2004); P. Wolf et al., Phys. Rev. Lett. 96, 060801 (2006); M. Romalis, in CPT and Lorentz Symmetry IV, Ref. [19]; V. A. Kostelecký and C. D. Lane, Phys. Rev. D 60, 116010 (1999); J. Math. Phys. (N.Y.) 40, 6245 (1999); C. D. Lane, Phys. Rev. D 72, 016005 (2005); D. Colladay and P. McDonald, Phys. Rev. D 73, 105006 (2006).

[26] R. Bluhm et al., Phys. Rev. Lett. 88, 090801 (2002); Phys. Rev. D 68, 125008 (2003).

[27] O. Bertolami et al., Phys. Lett. B 395, 178 (1997); G. Lambiase, Phys. Rev. D 72, 087702 (2005); J. M. Carmona, J.L. Cortés, A. Das, J. Gamboa, and F. Méndez, Mod. Phys. Lett. A 21, 883 (2006).

[28] H. Nguyen (KTeV Collaboration), in CPT and Lorentz Symmetry II, Ref. [19]; arXiv:hep-ex/0112046; A. Di Domenico (KLOE Collaboration), in CPT and Lorentz Symmetry IV, Ref. [19]; R. Ackerstaff et al. (OPAL Collaboration), Z. Phys. C 76, 401 (1997); M. Feindt et al. (DELPHI Collaboration), Report No. DELPHI 9798 CONF 80 (1997); K. Abe et al. (BELLE Collaboration), Phys. Rev. Lett. 86, 3228 (2001); B. Aubert et al. (BABAR Collaboration), Phys. Rev. Lett. 92, 142002 (2004); arXiv:hep-ex/0607103; arXiv: 0711.2713 [Phys. Rev. Lett. (to be published)]; J.M. Link et al. (FOCUS Collaboration), Phys. Lett. B 556, 7 
(2003); V. A. Kostelecký, Phys. Rev. Lett. 80, 1818 (1998); Phys. Rev. D 61, 016002 (1999); 64, 076001 (2001); N. Isgur et al., Phys. Lett. B 515, 333 (2001).

[29] G. W. Bennett et al. (g-2 Collaboration), Phys. Rev. Lett. 100, 091602 (2008); V. W. Hughes et al., Phys. Rev. Lett. 87, 111804 (2001); R. Bluhm et al., Phys. Rev. Lett. 84, 1098 (2000).

[30] L. B. Auerbach et al. (LSND Collaboration), Phys. Rev. D 72, 076004 (2005); M.D. Messier (SK), in CPT and Lorentz Symmetry III, Ref. [19]; B.J. Rebel and S.F. Mufson (MINOS), in CPT and Lorentz Symmetry IV, Ref. [19]; V. A. Kostelecký and M. Mewes, Phys. Rev. D 69, 016005 (2004); Phys. Rev. D 70, 031902(R) (2004); Phys. Rev. D 70, 076002 (2004); T. Katori, V. A. Kostelecký, and R. Tayloe, Phys. Rev. D 74, 105009 (2006); V. Barger, D. Marfatia, and K. Whisnant, Phys. Lett. B 653, 267 (2007).

[31] D. L. Anderson, M. Sher, and I. Turan, Phys. Rev. D 70, 016001 (2004); E. O. Iltan, Mod. Phys. Lett. A 19, 327 (2004).

[32] J. B. R. Battat, J. F. Chandler, and C. W. Stubbs, Phys. Rev. Lett. 99, 241103 (2007); H. Müller et al., Phys. Rev. Lett. 100, 031101 (2008); W. M. Jensen, S. M. Lewis, and J. C. Long, in CPT and Lorentz Symmetry IV, Ref. [19].

[33] Q. G. Bailey and V.A. Kostelecký, Phys. Rev. D 74, 045001 (2006).

[34] R. Utiyama, Phys. Rev. 101, 1597 (1956); T. W. B. Kibble, J. Math. Phys. (N.Y.) 2, 212 (1961).

[35] See, for example, R. Percacci, Nucl. Phys. B353, 271 (1991).

[36] V. A. Kostelecký and R. Potting, Gen. Relativ. Gravit. 37, 1675 (2005).

[37] M. Fierz, Helv. Phys. Acta 12, 3 (1939); M. Fierz and W. Pauli, Proc. R. Soc. A 173, 211 (1939).

[38] H. van Dam and M. Veltman, Nucl. Phys. B22, 397 (1970); V. I. Zakharov, JETP Lett. 12, 312 (1970).

[39] G. Yu. Bogoslovsky, SIGMAP Bulletin 1, 017 (2005); Phys. Lett. A 350, 5 (2006); X. Li and Z. Chang, arXiv:0711.1934v1.

[40] See, for example, J. W. Elliott, G. D. Moore, and H. Stoica, J. High Energy Phys. 08 (2005) 066; M. D. Seifert, Phys. Rev. D 76, 064002 (2007).

[41] See, for example, F. W. Hehl, P. von der Heyde, G. D. Kerlick, and J. M. Nester, Rev. Mod. Phys. 48, 393 (1976); I. L. Shapiro, Phys. Rep. 357, 113 (2002); V. A. Kostelecký, N. Russell, and J.D. Tasson, arXiv:
0712.4393 [Phys. Rev. Lett. (to be published)].

[42] C. M. Will and K. Nordtvedt, Astrophys. J. 177, 757 (1972); R. W. Hellings and K. Nordtvedt, Phys. Rev. D 7, 3593 (1973). See also C.M. Will, Theory and Experiment in Gravitational Physics (Cambridge University Press, Cambridge, 1993).

[43] V. A. Kostelecký and R. Lehnert, Phys. Rev. D 63, 065008 (2001).

[44] B. Altschul and V. A. Kostelecký, Phys. Lett. B 628, 106 (2005).

[45] C. Eling, T. Jacobson, and D. Mattingly, arXiv:gr-qc/ 0410001.

[46] C. Eling and T. Jacobson, Classical Quantum Gravity 23, 5643 (2006); C. Eling, Phys. Rev. D 73, 084026 (2006); B. Z. Foster, Phys. Rev. D 73, 104012 (2006).

[47] S. M. Carroll and E. A. Lim, Phys. Rev. D 70, 123525 (2004).

[48] B. M. Gripaios, J. High Energy Phys. 10 (2004) 069.

[49] M. L. Graesser, A. Jenkins, and M. B. Wise, Phys. Lett. B 613, 5 (2005).

[50] J.L. Chkareuli, C.D. Froggatt, and H.B. Nielsen, arXiv:hep-th/0610186.

[51] J.D. Bjorken, arXiv:hep-th/0111196; P. Kraus and E. T. Tomboulis, Phys. Rev. D 66, 045015 (2002); J. W. Moffat, Int. J. Mod. Phys. D 12, 1279 (2003); E. A. Lim, Phys. Rev. D 71, 063504 (2005); C. Heinicke, P. Baekler, and F. W. Hehl, Phys. Rev. D 72, 025012 (2005); O. Bertolami and J. Paramos, Phys. Rev. D 72, 044001 (2005); S. Kanno and J. Soda, Phys. Rev. D 74, 063505 (2006); L. Ackerman, S. M. Carroll, and M. B. Wise, Phys. Rev. D 75, 083502 (2007); J. L. Chkareuli, C. D. Froggatt, J. G. Jejelava, and H. B. Nielsen, arXiv:0710.3479; A. Halle and H. Zhao, arXiv:0711.0958.

[52] P. A. M. Dirac, Proc. R. Soc. A 209, 291 (1951); W. Heisenberg, Rev. Mod. Phys. 29, 269 (1957); P. G. O. Freund, Acta Phys. Austriaca 14, 445 (1961); J. D. Bjorken, Ann. Phys. (N.Y.) 24, 174 (1963).

[53] Y. Nambu, Prog. Theor. Phys. 190 (1968).

[54] M. A. Clayton, arXiv:gr-qc/0104103.

[55] V.A. Kostelecký, C. D. Lane, and A. G. M. Pickering, Phys. Rev. D 65, 056006 (2002); V. A. Kostelecký and A. G. M. Pickering, Phys. Rev. Lett. 91, 031801 (2003); G. de Berredo-Peixoto and I. L. Shapiro, Phys. Lett. B 642, 153 (2006); D. Colladay and P. McDonald, Phys. Rev. D 75, 105002 (2007); arXiv:0712.2055. 